\title{
Computational Aerodynamics Study of Competing Conceptual Designs for Advanced Tactical Fighter Aircraft
}

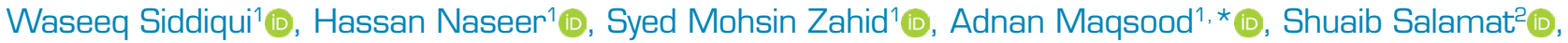 \\ Rizwan Riaz ${ }^{1}$ iD
}

1.National University of Sciences \& Technology - Research Center for Modeling \& Simulation - Computational Aeronautics Laboratory - Islamabad - Pakistan. 2. National University of Sciences \& Technology - College of Aeronautical Engineering Risalpur - Pakistan.

*Correspondence author: adnan@rcms.nust.edu.pk

\section{ABSTRACT}

Advanced tactical fighter (ATF) configurations are bound to perform high angle of attack (AoA) maneuvers. However, existing conceptual design tools available in aerospace industry are based on empirical or potential flows that cannot predict aerodynamic data in nonlinear regimes. High-fidelity computational fluid dynamics algorithms have to be incorporated during conceptual design phase for better assessment between competing configurations. In this research, steady state aerodynamic analysis is conducted to compare four conceptual designs of advanced tactical fighters through Reynolds-averaged Navier-Stokes (RANS) simulations. Prior to the study, two validation test cases were conducted based on ONERA M6 Wing and benchmark unmanned combat air vehicle (UCAV) design to assess the computational setup for the problem. Pressure based solver is used to model the flow field in subsonic, transonic and supersonic regimes at sea level for all four competing designs. The quantitative results include the aerodynamic forces and the longitudinal stability coefficient comparisons among the models and its components. The qualitative analyses include pressure distribution, eddy shedding and behavior of vortices at varying flow angle. Additionally, the empirical estimation for interpolation and post-stall extrapolation are carried out for further flight performance studies.

Keywords: Aircraft configurations; Static aerodynamic characteristic; Pitching moments.

\section{INTRODUCTION}

The conceptual stage of the aircraft design focuses on the efficient evaluation of various configurations. Due to time constraints, the experimental analysis in the form of flight and wind tunnel testing are not done at this stage. It takes considerable amount of time to construct a scaled down model that is geometrically similar to the intended configuration. Moreover, there is a significant experiment set up time that is not feasible for large volumes of configurations. Even when the testing begins, the probes, the wall and the sting effects affect the flow field that needs iterative corrections to eliminate interferences. Therefore, the analytical and computational tools are put to use for the rapid evaluation of the best performer in a series of different concepts economically. The linear regime of aerodynamics is studied mainly using empirical and linearized computer codes, which include the vortex lattice method (VLM), the potential flow theory and the lifting line theory (Schminder 2012; Amadori et al. 2008).

Received: Sept. 232020 | Accepted: Feb. 032021

Peer Review History: Double Blind Peer Review.

Section Editor: Paulo Greco 
The nonlinear phenomenon, which include the dominating viscous effects, are estimated using the codes VORSTAB (Tseng and Lan 1988) and DATCOM (Williams and Vukelich 1979). The VORSTAB code, which is based on the modifications to the potential flow theory, generates good estimates of stability coefficients that are in reasonable agreement with wind tunnel results. However, the code would provide successful prediction only when accurate inputs are provided, which include the geometrical representation of the model and the predicted location of the vortex lift. DATCOM, developed by United States Air Force (USAF) can provide rapid aerodynamic and stability assessment of simple shapes. The tool provides credible predictions of simple geometry over wide range of angles of attack (AoA), such as a body fin configuration (Abney and McDaniel 2005), but cannot estimate the aerodynamics of complex geometries and in transonic regime (Williams and Vukelich 1979). According to Blake (1985), digital DATCOM is not able to analyze the three-surface configurations (canard, wing, horizontal tail) in a single run.

Since the modern tactical aircraft has geometrical complexities and is expected to perform complex maneuvers, computational fluid dynamics (CFD) can be used as a decent alternative to experimentation for conceptual design analysis. As evident by its versatile use in Boeing (Tinoco 1991), CFD offers the capacity to solve a range of complicated flow problems where the empirical approach is inadequate, such as vortex dominated flows at high AoA. In the past, CFD faced several challenges in the conceptual design stage. The accuracy of the geometry in consideration was a major challenge for the designers. The absence of baseline for validation in the conceptual stage was an additional issue. Several aerodynamic performance indicators could not be assessed, such as the percentage of maximum leading-edge suction. These limitations created risks, especially for configurations that were designed to enter nonlinear flight regimes frequently (Mason et al. 1998). In addition, the computational cost and time of the CFD analysis was another concern. A lot of computational effort was required to perform meshing and simulation on one design. Today, the time of the cycle has decreased due to the evolution of the computing capacity and automated mesh generation algorithms. However, the amount of work needed in conventional conceptual design studies is still sufficiently high. Nevertheless, researchers and designers have started to rely on CFD for multidimensional problems due to rapidly increasing computational resources.

The Reynolds-averaged Navier-Stokes (RANS) based formulation has been widely used to study the external flow over aircraft shapes. Xue et al. (2016) investigated the characteristics of forward and backward sweep wings on aerodynamic characteristics. Zhong and Zhao (2012) learned the influence of engine nacelles and strakes on the maximum lift, the stall angle and the airflow around civil aircraft at high angle of attack (AoA). For a light aircraft configuration, Kostić et al. (2014) performed and compared CFD results against DATCOM and VLM. Similarly, Nicolosi et al. (2014) studied the aerodynamic interference due to sideslip on aircraft parts and the behavior of aerodynamic coefficients on various fuselage shapes of large turbo propeller aircraft. The evaluation of stability derivatives through CFD has also attracted appreciable attention in the past. Kryvokhatko and Masko (2017) employed the use of CFD method to study the longitudinal static stability and aerodynamic characteristics of a tube launched unmanned aerial vehicle (UAV) with variable wings rotation angles. Similarly, the static stability and neutral point was investigated in the work of Bitencourt et al. (2011). Schminder (2012) investigated the influence of canard and its horizontal location relative to center of gravity (CG) numerically and experimentally through wind tunnel test. It was observed that the presence of canard leads to instability of the aircraft while also changes the trim angle, elevates the maximum lift coefficient and delays stall point.

In CFD, it is essential to model or resolve turbulence. Turbulent flows are commonly calculated using RANS models. These models include SST k- $\omega$ (Menter 1994) and Spalart-Allmaras (Spalart and Allmaras 1992). While RANS is widely used for credible estimates with least computational cost, higher fidelity tools become a necessity to resolve eddies for detailed flow physics, such as aggressive flow separations. These techniques include large eddy simulation (LES), hybrid RANS-LES and the very expensive direct numerical simulation (DNS). Details on these models can be found in the Ansys Fluent Theory Guide (ANSYS 2017).

While RANS turbulence models have had a lot of success in a variety of applications, the accurate prediction of some complex flow phenomena such as 3D flow separation necessitates an experimental data or validation study to calibrate and finalize a turbulence model for an application. Della Vecchia et al. (2013) used the Spalart-Allmaras model to test the DLR-F11 configuration and found it to be suitable for predicting the stall point. Similarly, SST k- $\omega$ model was proven to perform best among the one and two equation eddy viscosity models for supersonic rocket aerodynamics (López et al. 2013).

Tactical fighter aircraft offers complicated flow physics at high AoA. Boelens (2012) studied the formation of several vortices on the X-31 aircraft for AoA greater than $12^{\circ}$. These vortices originated from different components of the model, which included canard, 
fuselage and inlet region. Recent studies (Wibowo et al. 2018; Sutrisno et al. 2019) have studied the detection of vortex phenomena. While higher fidelity tools like detached eddy simulation are widely accepted to accurately capture these complex phenomena, the model SST k- $\omega$ can fairly produce credible information about the flow as it succeeds in detecting the vortex breakdown location at higher AoA.

The present study evaluates the aerodynamic and static longitudinal stability of four different aircraft geometries using the Navier-Stokes formulation. The aerodynamic coefficients are iteratively calculated through CFD runs on a wide range of angles and flow velocities. Moreover, the post-stall stability characteristics are determined and compared among the configurations. The main objective of this research is to perform a comparative analysis of different aerodynamic configurations over the range of parameters through high fidelity simulations and develop better estimations in the conceptual phase. To optimize the design, it is essential to analyze the drag, lift and static stability trends of the fuselage, canards, wings and tails. This study also analyzes the aerodynamic characteristics of the components.

In this article, the section "Problem Formulation" covers the problem formulation, validation cases and the geometric modeling of the configurations. The quantitative and qualitative results are then discussed in the section "Results and Discussions", which includes the aerodynamic performance plots, pressure contours and flow vortices. The analytical estimations made through Polhamus method and post-stall aerodynamic predictions are also carried out. Finally, the conclusions drawn from the study are highlighted in the end.

\section{Problem Formulation}

The computational setup for this research was created by using three-dimensional RANS equations and pressure-based solver. Steady state solution was calculated using the finite volume method based commercial solver Ansys Fluent on unstructured computational grids comprising of tetrahedral, triangle, prism and pyramid elements. The comparative study of the configurations begins with the validation of test cases. Since no experimental data was available for models under consideration, some reference studies were highly necessary to validate the computational approach. Therefore, two validation studies were conducted based on a sweptback wing and an unmanned combat aerial vehicle (UCAV). Upon validation of the computational framework, four aircraft concepts were modeled and simulated. In the present research work, the grids were generated using Ansys Meshing and ICEM CFD. Numerical solution was performed in Ansys Fluent and the postprocessing of the results was performed using Tecplot, Ansys CFD post and MATLAB.

\section{Validation Study on ONERA M6 Wing}

A three-dimensional computational flow study is considered on the semispan swept wing ONERA M6 wing (Fig. 1). The test case is widely popular for validation for external flows in transonic regime. The computational domain of the model is a bullet-shaped domain composing of pressure far field surfaces and a symmetric plane. The dimensions of the domain are also presented in Fig. 1. Due to the symmetric nature of the problem, only half geometry is modeled along the XZ plane to reduce the computation time.

(a)

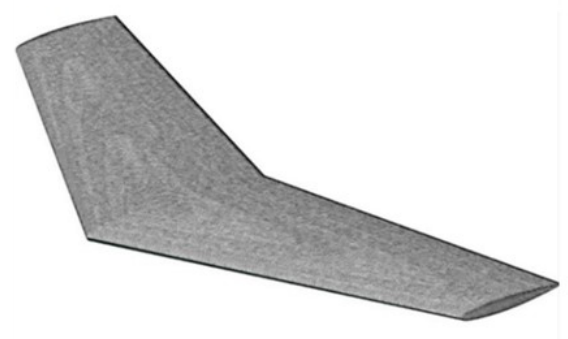

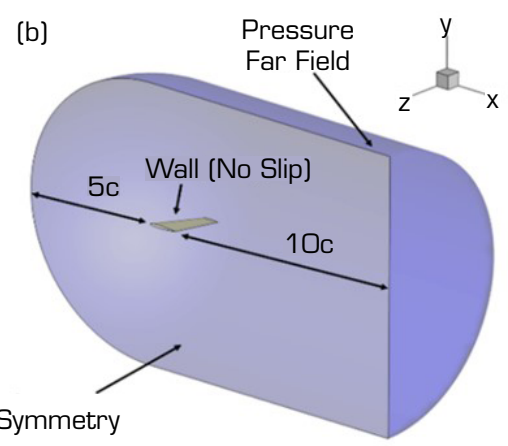

Figure 1. Computational Setup of ONERA M6 wing. (a) Geometric illustration; (b) Computational domain.

The validation test case is performed using a steady state pressure-based solver. Simple scheme is selected with least square cell based gradient and second order upwind for the remaining equations. SST k- $\omega$ is chosen as the turbulence model in this validation case under the following flow simulation conditions: Mach 0.8395 , angle $3.06^{\circ}$, static temperature $255.56 \mathrm{~K}$ and gauge pressure 45.829 psi. The M6 wing is meshed using Ansys ICEM CFD and Ansys Meshing. Unstructured computational grids comprising of 
tetrahedral and prism volume and triangle surface elements are generated. With the expansion ratio set to 1.2, 30 inflation layers are placed and initial height is adjusted to reach $\mathrm{y}^{+}$below 1 . The final grid sizes are 4.66 million elements for the Ansys ICEM CFD grid and 4.92 million elements for the Ansys Meshing. These grids are simulated and the numerical results are compared to the experimental data of pressure coefficient distribution from the analysis by Schmitt and Charpin (1979). The findings are plotted on different cross sections of the wing in the form of a pressure coefficient plot against the normalized chord length. As visible in Fig. 2, a good agreement between the numerical and experimental results is observed.

(a)

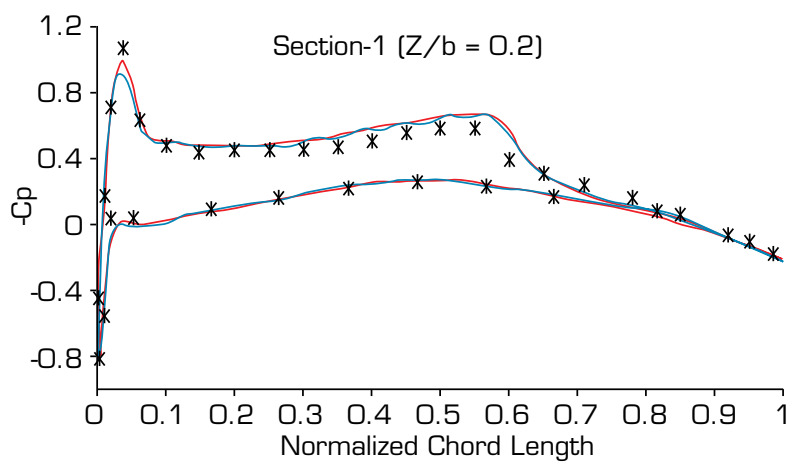

(c)

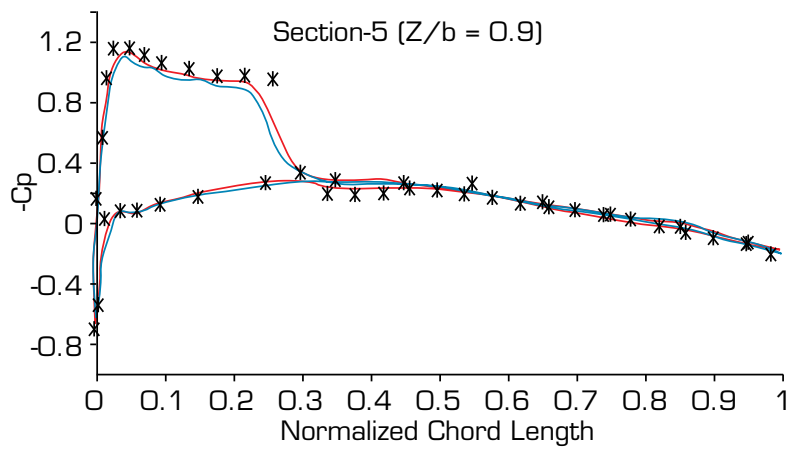

(b)

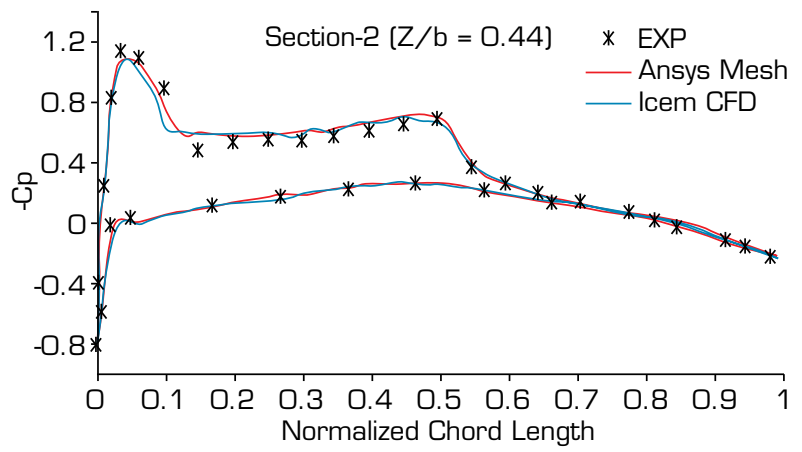

(d)

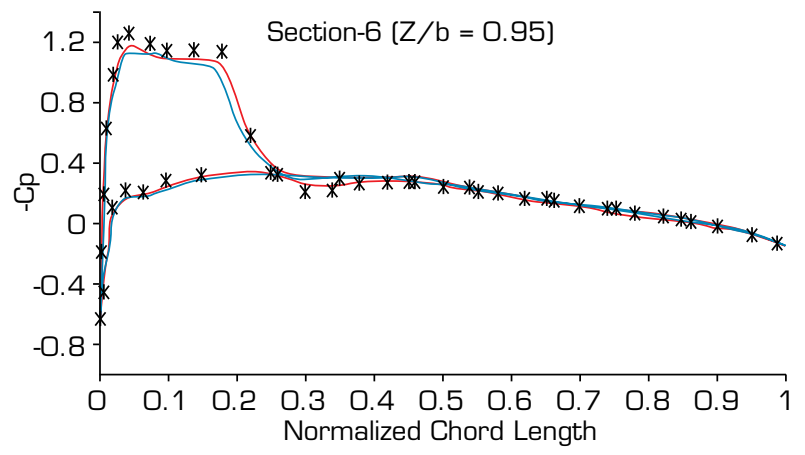

Figure 2. Pressure coefficient distribution at various sections of the wing. (a) Section $1(\mathrm{Z} / \mathrm{b}=0.2)$;

(b) Section $2(\mathrm{Z} / \mathrm{b}=0.44)$; (c) Section $5(\mathrm{Z} / \mathrm{b}=0.9)$; (d) Section $6(\mathrm{Z} / \mathrm{b}=0.95)$.

Additionally, the effect of nondimensional first cell distance $\mathrm{y}^{+}$is investigated on the chosen turbulence model. Three different grids comprising of $\mathrm{y}^{+}$less than 1, less than 30 and less than 100 are investigated. It is found that the coarsest grid ( $\mathrm{y}^{+}$less than 100) has the maximum percentage difference of $6.72 \%$ with reference to the finest grid that can be considered insignificant. Computational results for the opted range of $\mathrm{y}^{+}$are well coherent with experimental data allowing the flexibility of numerical grid, as can be seen in Fig. 3. However, the recommended range of $\mathrm{y}^{+}$for SST $\mathrm{k}-\omega$ turbulence model is $0<\mathrm{y}^{+}<1$.
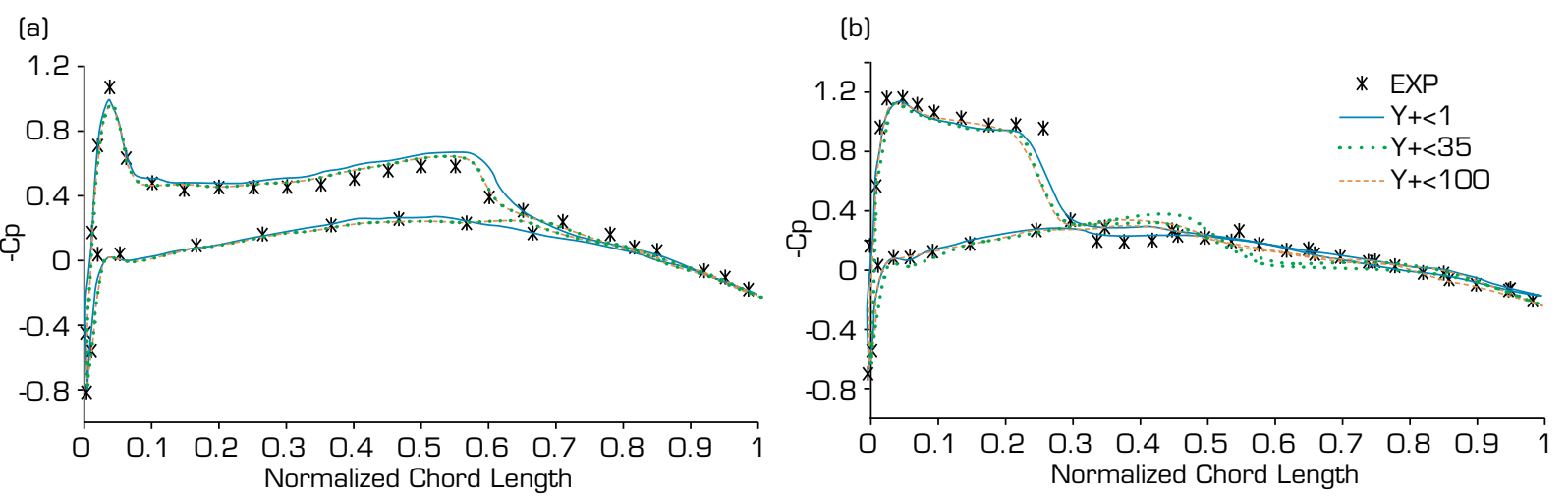

Figure 3. Influence of $\mathrm{y}^{+}$on pressure coefficient distribution of the wing. (a) Section $1(\mathrm{Z} / \mathrm{b}=0.2)$; (b) Section $5(\mathrm{Z} / \mathrm{b}=0.9)$. 


\section{Validation Study on UCAV}

A low subsonic and incompressible fluid analysis is computationally conducted for the model UCAV. The stability and control configuration (SACCON) model was designed by the members of the AVT-161 task group on applied vehicle technology to provide a platform for static and dynamic experimental measurements, as well as for various computational simulations. With a leadingedge sweep angle of $53^{\circ}$, the model has a lambda wing platform. The root chord is around $1 \mathrm{~m}$ and the wing span is $1.53 \mathrm{~m}$, while $0.479 \mathrm{~m}$ is the mean aerodynamic chord and $0.77 \mathrm{~m}^{2}$ is the reference wing area (Schütte et al. 2009). Based on the computational and experimental work (Loeser et al. 2010), a geometrically similar model is constructed and simulated for assessing the credibility of the turbulence model SST k- $\omega$.

The computational domain of the present study is similar in terms of geometrical dimensions to the previous validation test case. Since the flow is incompressible, inlet surfaces of the domain are modeled as velocity inlet, where the static temperature, flow direction and the velocity magnitude are assigned. Pressure outlet condition is imposed on the surface downstream of the wing, where atmospheric static pressure and total temperature are assigned.

The resulting grid, composing of roughly 4.5 million elements of half modeled UCAV, is simulated at sea level on a wide range of AoA. A comparison of drag coefficient $C_{D}$ is plotted against the AoA in Fig. 4 between the experimental and numerical study of the present research. It can be observed that the turbulence model closely matches the experimental data till $22^{\circ}$. The coefficient of drag is still within $10 \%$ of the experimental work beyond $22^{\circ}$, which can be considered acceptable. The numerical results again match decently near $30^{\circ}$ with the experiment. The pressure distribution on the UCAV is shown in Fig. 5.

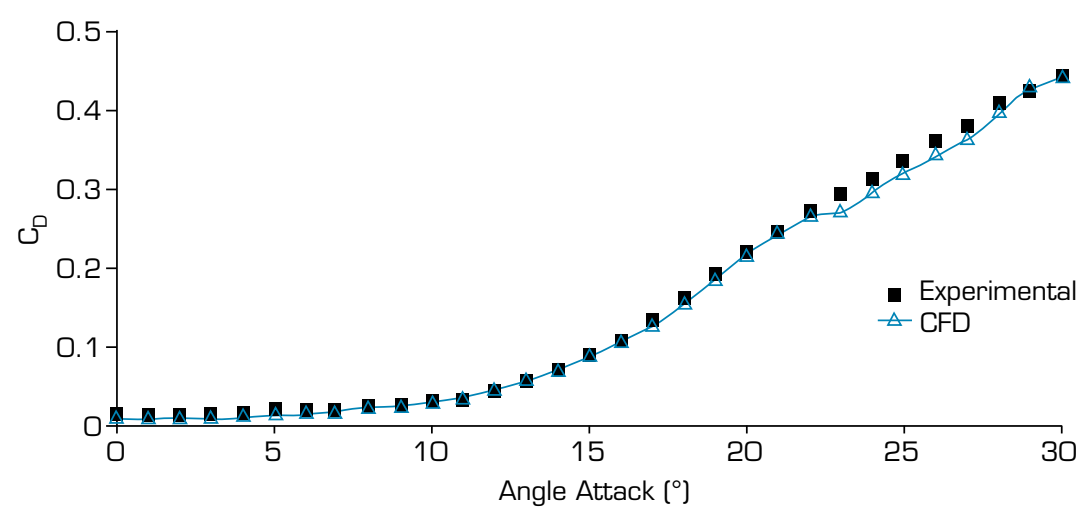

Figure 4. Comparison of $C_{D}$ vs. AoA of CFD analysis with experimental data (Loeser et al. 2010).

(a)

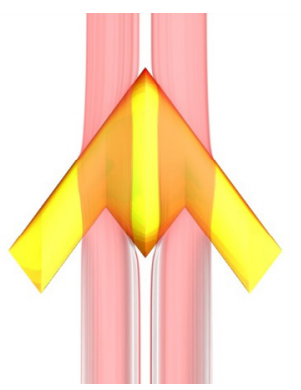

(b)

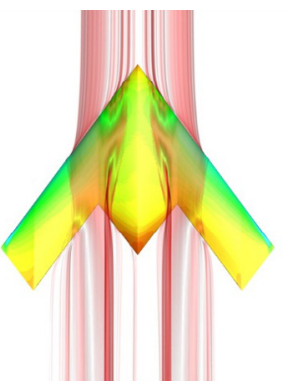

(c)

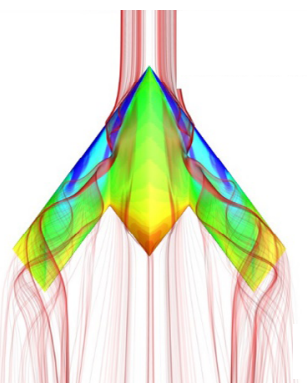

Pressure Coefficient (Cp)

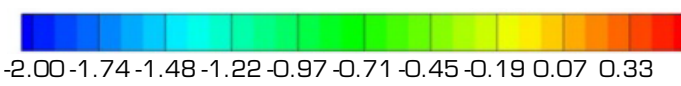

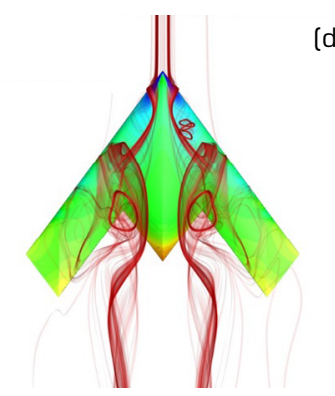

(d)

Figure 5. Flow visualization and $\mathrm{C}_{\mathrm{p}}$ contours on upper surface of UCAV. (a) $0^{\circ}$ AoA; (b) $10^{\circ}$ AoA; (c) $20^{\circ}$ AoA; (d) $30^{\circ}$ AoA. 


\section{Modeling and Simulation of Conceptual Configurations}

Computational modeling of subject study is followed by the development of accurate geometries of competing designs. Name convention for conceptual designs is set as concept-X (C-X), where X ranges from 0 to 3. Computer-aided design (CAD) models of airframes (Fig. 6) were developed in SolidWorks. Concept-0 is about V-tail idea to reduce structural weight. Concept-1 uses conventional setup of vertical and horizontal stabilizers with modified airframes. Canards are introduced in C-2 and C-3 as an effective pitch control mechanism. The geometrical dimensions are presented in Fig. 7 and Table 1.

(a)

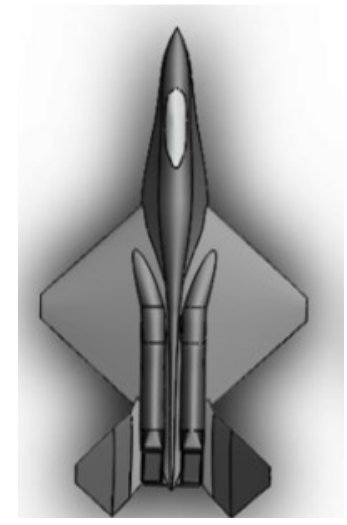

(b)

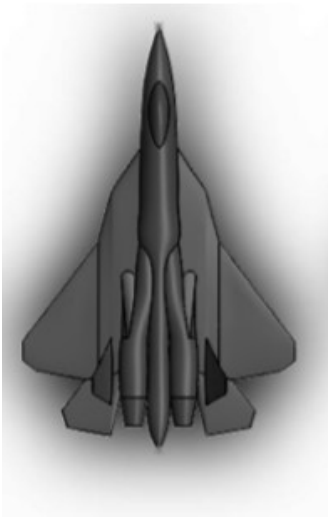

(c)

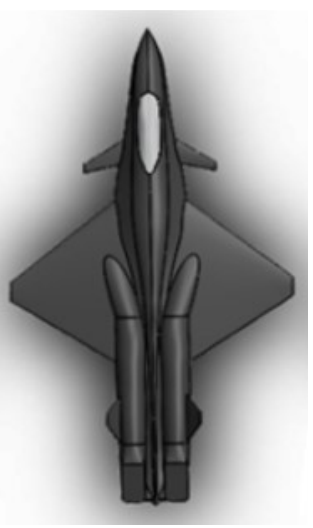

(d)

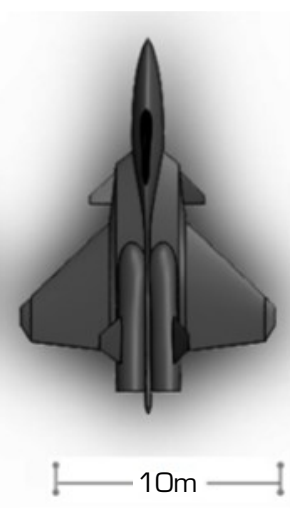

Figure 6. Computer-aided design models of all the configuration. (a) Concept-0; (b) Concept-1; (c) Concept-2; (d) Concept-3.

(a)
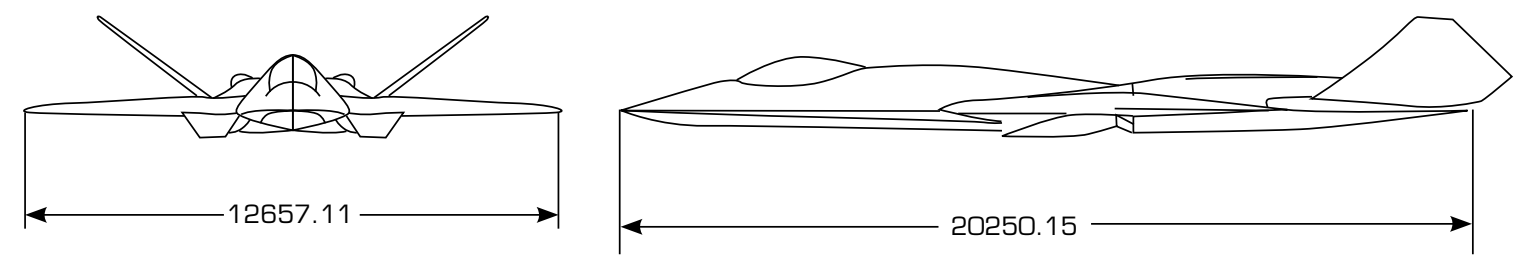

(b)
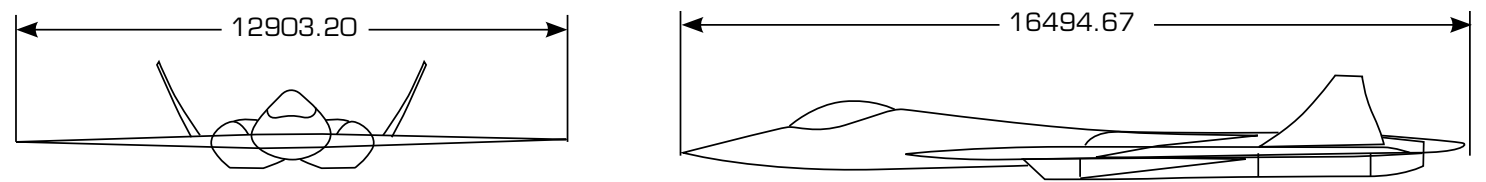

(c)
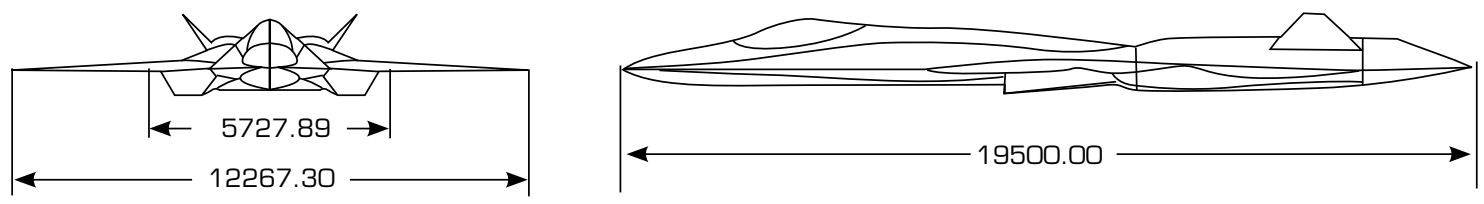

(d)
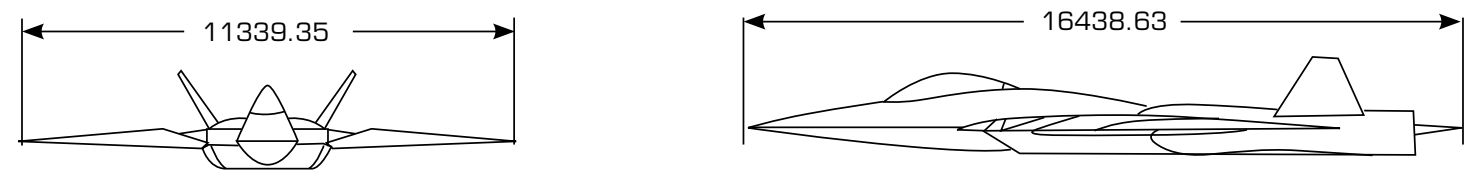

Figure 7. Geometric Dimensions of the configurations. (a) Concept-0; (b) Concept-1; (c) Concept-2; (d) Concept-3.

Table 1. Detailed dimensions of the models. 


\begin{tabular}{|c|c|c|c|c|}
\hline \multirow{2}{*}{ Parameters } & \multicolumn{4}{|c|}{ Values/Details } \\
\hline & C-O & C1 & $\mathrm{C2}$ & C3 \\
\hline Fuselage Length & $20.25 m$ & 18.49. & $19.50 \mathrm{~m}$ & $16,44 m$ \\
\hline AircraftSpam & $12.66 \mathrm{~m}$ & $12.90 \mathrm{~m}$ & $12.27 \mathrm{~m}$ & $11.34 \mathrm{~m}$ \\
\hline Aspect Ratio & 2.00 & 2.31 & 2.51 & 2.52 \\
\hline Wing Leading Edge Sweep & $40.00^{\circ}$ & $50.64^{\circ}$ & $34.67^{\circ}$ & $43.00^{\circ}$ \\
\hline Vertical Tail Leading Edge Sweep & $47.00^{\circ}$ & $45.00^{\circ}$ & $46.65^{\circ}$ & $25.00^{\circ}$ \\
\hline Horizontal Tail Leading Edge Sweep & - & $50.20^{\circ}$ & - & - \\
\hline Camard Sweep & - & - & $30.00^{\circ}$ & $44.00^{\circ}$ \\
\hline Wing Airfoil & NACA 65-206 & NACA 65-206 & NACA 65-206 & NACA 65-206 \\
\hline Horizontal Tail/ Canard Airfoil & NACA 0006 & NACA 0006 & NACA 0006 & NACA 0006 \\
\hline Reference Area of Vertical Tail & $13.83 \mathrm{~m}^{2}$ & $8.48 m^{2}$ & $10.12 \mathrm{~m}^{2}$ & $5.62 m^{2}$ \\
\hline Reference Area of Wing & $80.00 m^{2}$ & $72.00 \mathrm{~m}^{2}$ & $60.00 \mathrm{~m}^{2}$ & $51.00 \mathrm{~m}^{2}$ \\
\hline Reference Area of Canard & - & - & $5.06 \mathrm{~m}^{2}$ & $2.86 \mathrm{~m}^{2}$ \\
\hline Reference Area of HorizontalTail & - & $13.20 \mathrm{~m}^{2}$ & - & - \\
\hline Span of Horizontal Tail & - & $9.14 \mathrm{~m}$ & - & - \\
\hline Span of Canard & - & - & $5.73 m$ & $5.12 \mathrm{~m}$ \\
\hline Span of Wing & $12.66 \mathrm{~m}$ & $12.90 \mathrm{~m}$ & $12.27 \mathrm{~m}$ & $11.34 \mathrm{~m}$ \\
\hline Mean Aerodynamic Chord of Wing & $8.99 m$ & $8.03 \mathrm{~m}$ & $6.04 \mathrm{~m}$ & $7.39 \mathrm{~m}$ \\
\hline Mean Aerodynamic Chord of Horizontal Tail & - & $3.86 m$ & - & - \\
\hline Mean Aerodynamic Chord of Vertical Tail & $4.17 \mathrm{~m}$ & $2.08 \mathrm{~m}$ & $2.37 \mathrm{~m}$ & $1.47 \mathrm{~m}$ \\
\hline Mean Aerodynamic Chord of Canard & - & - & $2.02 \mathrm{~m}$ & $1.67 \mathrm{~m}$ \\
\hline Root Chord of Wing & $11.62 \mathrm{~m}$ & $10.22 \mathrm{~m}$ & $9.00 \mathrm{~m}$ & $9.37 \mathrm{~m}$ \\
\hline Root Chord of Horizontal Tail & - & $2.69 \mathrm{~m}$ & - & - \\
\hline Root chord of Vertical Tail & $4.34 \mathrm{~m}$ & $2.92 m$ & $2.42 \mathrm{~m}$ & $2.06 \mathrm{~m}$ \\
\hline Root Chord of Canard & - & - & $1.86 \mathrm{~m}$ & $1.45 \mathrm{~m}$ \\
\hline Tip chord of Wing & $1.02 \mathrm{~m}$ & $0.84 \mathrm{~m}$ & $0.76 \mathrm{~m}$ & $0.79 \mathrm{~m}$ \\
\hline Tip Chord of Horizontal Tail & - & $0.80 \mathrm{~m}$ & - & - \\
\hline Tip Chord of Vertical Tail & $0.80 \mathrm{~m}$ & $0.71 \mathrm{~m}$ & $0.47 \mathrm{~m}$ & $0.60 \mathrm{~m}$ \\
\hline Tip Chord of Camard & - & - & $0.46 \mathrm{~m}$ & $0.33 \mathrm{~m}$ \\
\hline
\end{tabular}

The concepts were modelled based on transonic area rule. Transonic area rule is an effective design technique used to reduce wave drag in transonic flight. It addresses the smooth distribution of longitudinal cross-sectional area of aircraft usually by "wasting" fuselage near wings. Longitudinal cross-sectional area distribution is presented by Fig. 8 for all the configurations. Sharp edges showing the abrupt changes in area distribution for C- 0 and C-2 make these airframes more vulnerable to the formation of strong shock wave and consequent drag rise in transonic flight. 


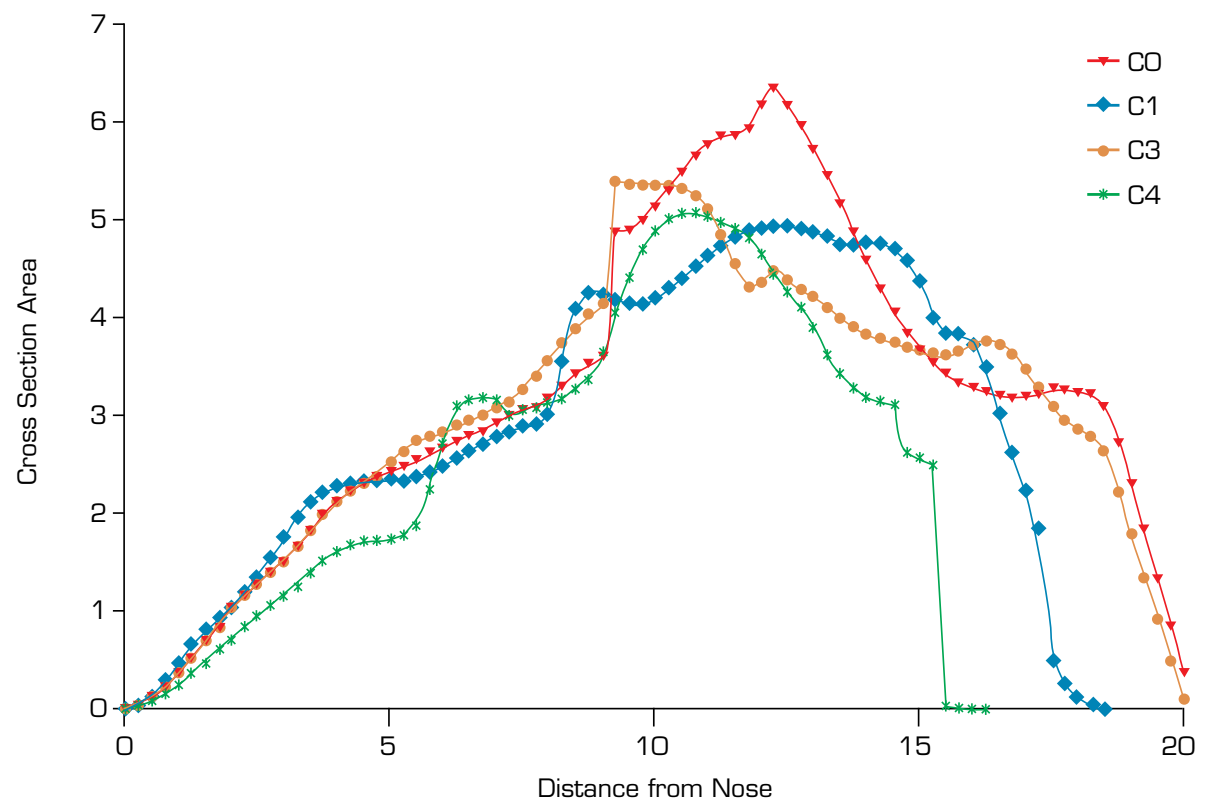

Figure 8. Longitudinal cross-sectional area distribution for area rule estimation (distance in $m$ and area in $m^{2}$ ).

The preprocessing and simulation setup for the models follows the same methodology as adopted in the validation test cases. The domain for each configuration is set similarly to the validation studies (Fig. 1), where the chord would now represent the fuselage length of the aircraft. The boundary conditions are set as pressure far field for the external surfaces of the domain and symmetry condition for the symmetric plane of the domain. While the wall condition is imposed on the aircraft, the duct intake surface is assigned velocity inlet condition for subsonic stall study and no-slip condition for the remaining analysis. Fifteen layers of the nodes are placed in the boundary layer over the refined surface mesh for exhibiting average value of $y^{+}$less than 100 . The grid independence of configurations is conducted on the basis of the coefficient of drag and lift. Based on the results, similar meshing parameters are assigned to the remaining models for comparative analysis. The final grid sizes for a particular model poses 7 to 9 million elements. A representation of the volume and surface mesh is illustrated in Fig. 9.

(a)

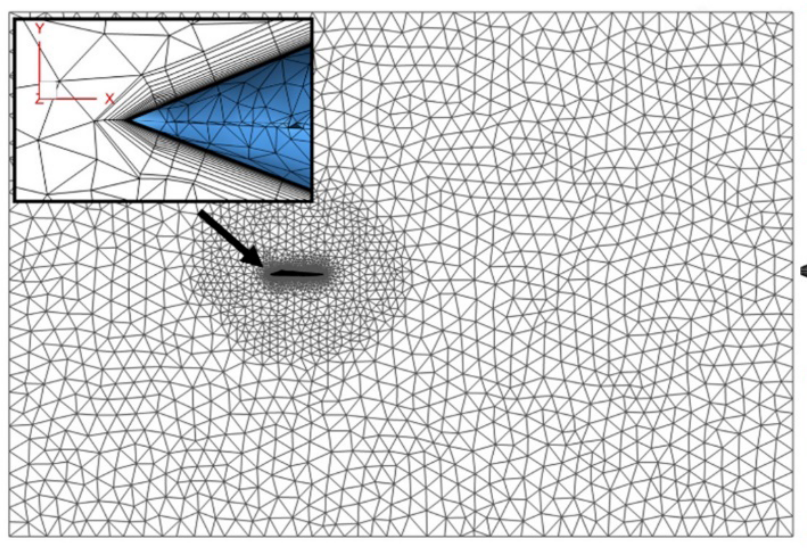

(b)

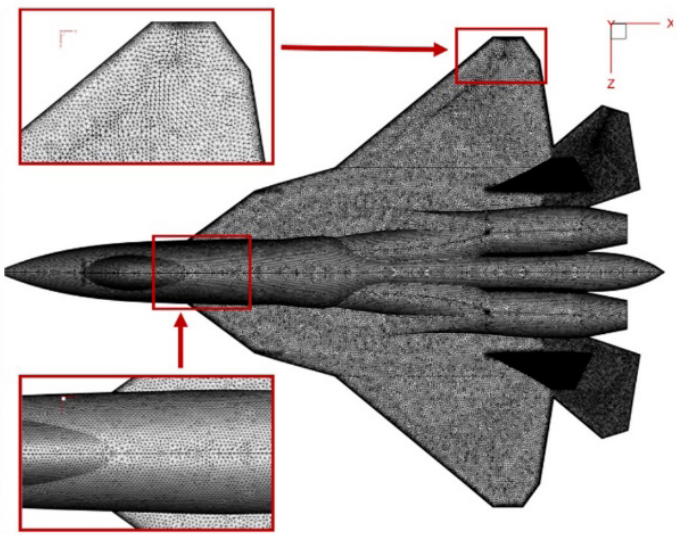

Figure 9. Meshing Topology of C-1 (a) Volume Mesh; (b) Surface Mesh.

The solution method for all simulations is set to the pressure-velocity coupled scheme. SST k- $\omega$ is employed as the turbulence model for complete analysis. The pressure, density, momentum, turbulent kinetic energy, specific dissipation rate and energy are all set to second order upwind. Viscosity is calculated using Sutherland's law and the density is set to ideal gas. The aerodynamic coefficients are monitored under the convergence criteria $10^{-6}$. The grids are simulated under the flow conditions summarized in Table 2 . 
Table 2. Flow conditions for the study.

\begin{tabular}{cc}
\hline Altitude & Sea level \\
\hline Gauge pressure $(\mathrm{Pa})$ & $101325.0 \mathrm{~Pa}$ \\
\hline Static temperature & $300.0 \mathrm{~K}$ \\
\hline Subsonic Mach No. & 0.6 \\
\hline Supersonic Mach No. & 1.4 \\
\hline Subsonic AoA range & -10.0 to $60.0^{\circ}$ \\
\hline Supersonic AoA range & -8.0 to $20.0^{\circ}$ \\
\hline Mach range for zero-lift drag & 0.2 to 2.0 \\
\hline
\end{tabular}

Approximately 360 simulations are performed in this study. In order to accelerate the computation, parallel processing via grid partitioning and load balancing on multiple compute nodes is performed on each computational domain.

\section{RESULTS AND DISCUSSIONS}

\section{Aerodynamic Forces and Moment Analysis}

Aerodynamic evaluation is led by the comparison of basic aerodynamic coefficients, that is, the zero-lift drag coefficient $\mathrm{C}_{\mathrm{Do}}$, drag coefficient $C_{D}$, lift coefficient $C_{L}$ and lift-drag ratio $\mathrm{L} / \mathrm{D}$ characteristics between the competing configurations.

The zero-lift drag coefficient, $\mathrm{C}_{\mathrm{Do}}$, is an important parameter to determine the aerodynamic efficiency of the airframe. It mainly relates the wetted area of aircraft with the skin friction drag and pressure drag. Conventionally, the zero-lift drag radically increases in transonic region because of the shock wave formation. Due to its significant effect on aircraft overall size and performance, it is imperative to accurately estimate the $\mathrm{C}_{\mathrm{Do}}$ even in the preliminary design stage. Figure 10 is depicting the variation in zero-lift drag coefficient at corresponding zero-lift AoA $\left(\alpha_{0}\right)$ of each conceptual design in subsonic, transonic and supersonic regimes. Concept-1 and C-2 represent the minimum and maximum value of zero-lift drag throughout the Mach range, respectively. Transonic region is highlighted in the reference Fig. 8 , indicating the lowest and highest transonic drag rise for C-1 and C-2 respectively. Zero-lift drag coefficient $\mathrm{C}_{\mathrm{Do}}$ vary almost alike till Mach 1 for C-0 and C-3 and rises for C-3 configuration in supersonic region. After the transonic region, drag appears to drop from maximum value in a benign manner. These observations are in coherence with the implications made through transonic area rule estimation (Fig. 8).

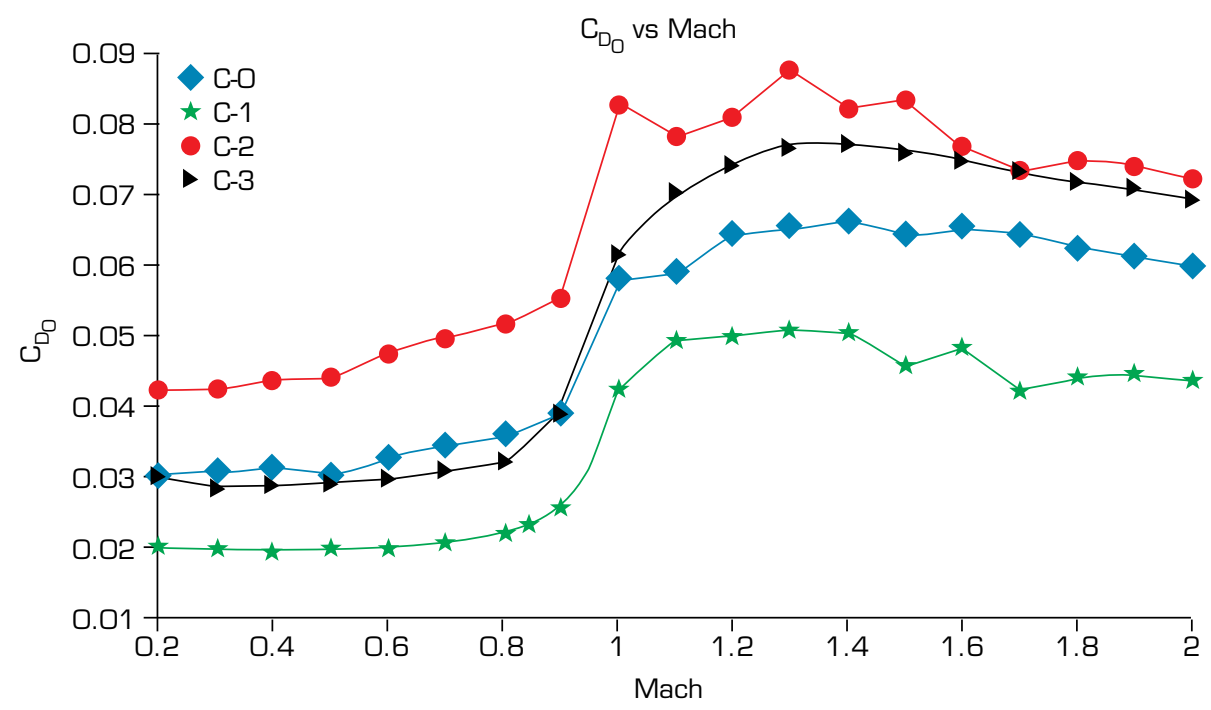

Figure 10. Zero-lift drag coefficient vs. Mach number. 
Lift and drag characteristics are evaluated through their respective coefficients. Figure 11 shows the aerodynamic characteristics of concepts at subsonic and supersonic Mach. It can be observed that the lift and drag coefficients vary conventionally and are alike in the subsonic regime for all the configurations. Fairly straight slope of lift coefficient can be seen for all the configurations till $12^{\circ}$ AoA. The highest value of lift coefficients in ascending order can be observed for C-0, C-1, C-2 and C-3 at $20^{\circ}$ AoA. The approximate subsonic stall angle for all the configurations is $25^{\circ}$.

(a)

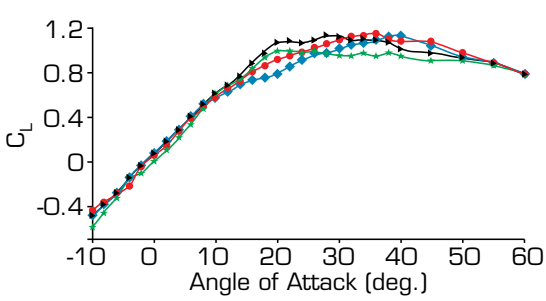

(d)

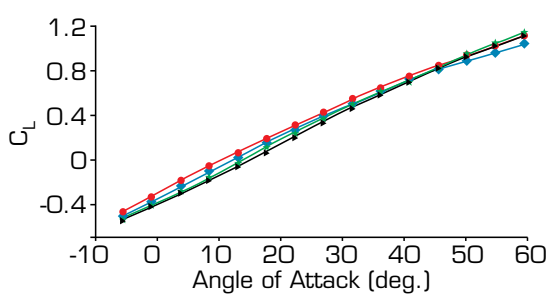

(b)

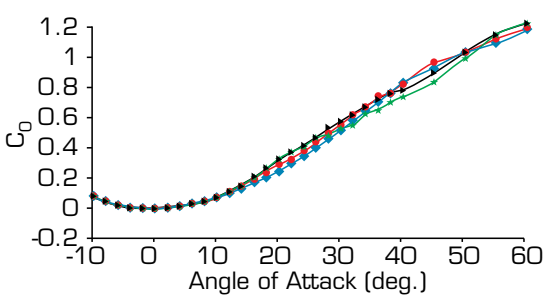

(e)

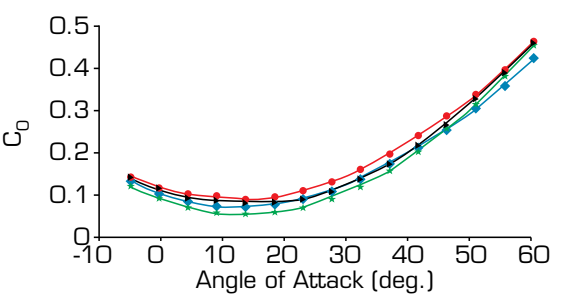

(c)

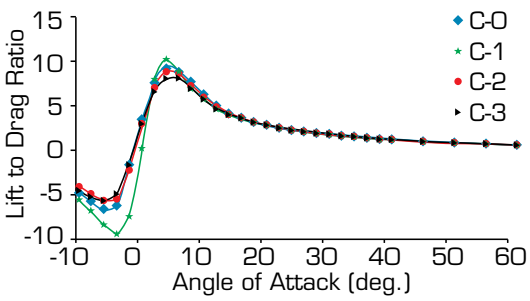

(f)

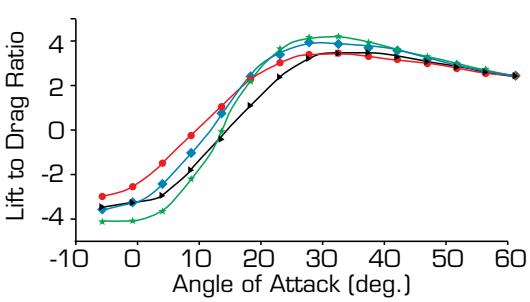

Figure 11. Comparison of aerodynamic performance at subsonic and supersonic Mach. (a) Subsonic $C_{L}$ vs. AoA; (b) Subsonic $C_{D}$ vs. AoA; (c) Subsonic L/D vs. AoA; (d) Supersonic $C_{L}$ vs. AoA; (e) Supersonic $C_{D}$ vs. AoA; (f) Supersonic L/D vs. AoA.

A flat surface behavior can be seen beyond $50^{\circ}$ AoA. The general trend with the increasing AoA is consistent for all the airframes. Concept-2 has highest supersonic lift coefficient with the penalty of highest supersonic drag coefficient. At lower AoA, C-0 has comparatively better lift coefficient with moderate values of drag coefficient till $14^{\circ}$ AoA. At higher AoA, highest value of supersonic lift and drag coefficients can be observed for C-1 configuration. Concept-1 has the advantage for the minimum value of supersonic drag coefficient at lower AoA, as highlighted in Fig. 11.

The overriding concern in designing the airframe is to achieve the maximum lift-to-drag ratio. The overall trend of all the configurations is consistent. The maxima of subsonic L/D for all the configurations ranges from 8 to 10 , which can be observed at $4^{\circ} \mathrm{AoA}$, as highlighted in Fig. 11. The maxima of supersonic L/D for all the configurations ranges from 3 to 4 , which lies in between 6 and $8^{\circ}$ AoA. Concept- 1 is showing the highest lift-to-drag ratio among all the configurations in both Mach regimes.

In preliminary design study of the aircraft, one of the major stability concerns is to ensure inherited longitudinal static stability of the airframe. Pitching attitude is a key indicator to evaluate the longitudinal static stability of the aircraft. It is highly dependent on aircraft center of gravity $(C G)$ location $\left(X_{C G}\right)$ and determined by the pitching moment coefficient $\left(C_{m}\right)$. The concept of neutral point $\left(X_{N P}\right)$ or the location of aircraft aerodynamic center can be regarded as an alternative stability criterion. Therefore, neutral point variation study is imperative in defining the longitudinal static stability and CG positioning of the aircraft. The pitching moment coefficient at zero AoA $\left(C_{m_{o}}\right)$ has to be positive to get adequate lift to balance the weight of the aircraft. The slope of $C_{m}$-alpha curve can be negative, zero or positive depending on whether $X_{C G}$ is less than, equal to or greater than $X_{N P}$ respectively. For the longitudinal static stability, slope of $C_{m}$-alpha curve should be negative or the location of center of gravity should lie forward of the neutral point. For quantifying the degree of static pitching stability, stick-fixed static margin is used and expressed through the formulation Eq. 1 from (Nelson 1998):

$$
S M_{\text {stick-fixed }}=\frac{X_{N P}}{\bar{c}}-\frac{X_{C G}}{\bar{c}}=-\frac{C_{m_{\alpha}}}{C_{L_{\alpha}}}
$$


The pitching moment coefficient at various longitudinal positions are computed to identify the neutral point of the aircraft. Center of gravity locations of each configuration, their neutral points and respective stick-fixed static margin in both subsonic and supersonic Mach regimes are tabulated in Table 3. Concept- 0 is the only configuration with positive stick-fixed static margin at supersonic speed. The rest of the configurations have negative stick-fixed static margin in both Mach regimes, which implies that their CGs lie aft of the neutral point and the neutral point variation with the increasing Mach is insignificant.

Table 3. Neutral point variation and respective stick-fixed static margins of all the configurations.

\begin{tabular}{|c|c|c|c|c|c|}
\hline \multirow{2}{*}{$\begin{array}{l}\text { Conceptual } \\
\text { design }\end{array}$} & \multirow{2}{*}{$\begin{array}{l}\text { CG location from nose } \\
\qquad X_{c g}(\mathrm{~m})\end{array}$} & \multicolumn{2}{|c|}{ Neutral point from nose $X_{N P}(\mathrm{~m})$} & \multicolumn{2}{|c|}{ Stick-fixed static margin } \\
\hline & & Subsonic & Supersonic & Subsonic & Supersonic \\
\hline $\mathrm{C}-\mathrm{O}$ & 12.06 & 11.08 & 12.50 & -0.11 & 0.04 \\
\hline C-1 & 13.94 & 10.95 & 12.00 & -0.36 & -0.23 \\
\hline C-2 & 10.86 & 8.90 & 9.86 & -0.19 & -0.13 \\
\hline C-3 & 11.93 & 9.60 & 10.40 & -0.37 & -0.24 \\
\hline
\end{tabular}

The subsonic pitching attitude at various longitudinal positions for each configuration with the behavior change in static stability experienced by shifting the location of CG is shown in Fig. 12. The displacement in the aft direction and forward direction is represented by a positive and negative sign, respectively. It is evident from the figure that all configurations are longitudinally statically unstable in subsonic regime and exhibit nonflyable value of $C_{m_{o}}$ (i.e., $\leq 0$ ). However, the overall stability is improved at supersonic speed as the neutral point shifts almost to half chord at supersonic state. Comparatively, C-0 is quite closer to the stable configuration as its $C_{m}$-alpha curves are relatively less steep. Adequately positive values of $C_{m_{o}}$ for C-1 are making it fly-worthy. The rest of the configurations are almost consistent in supersonic pitching attitude.

\section{Flow Physics}

The contours of pressure coefficient $\left(\mathrm{C}_{\mathrm{p}}\right)$ at different AoA are plotted in both subsonic and supersonic regimes at different flow angles in Fig. 13. By comparing the pressure distribution on the upper surface of the conceptual aircraft at Mach 0.6 and $8^{\circ}$ AoA, it can be seen that the highest negative pressure is found over the leading edge of C-2 aircraft. Concept- 0 has uniform pressure distribution over the wing surface. The same can also be observed over C-2 and C-3 aircraft with small span wise component. Uniformity is not maintained over the $\mathrm{C}-1$, mainly due to the wing tip vortices. Concept-1 has the best pressure distribution over the blended engine nacelle, primarily due to superior fairing than other aircraft, while C-3 shows high pressure regions and thus suggests improvements for engine nacelle shape. The canards are only in C-2 and C-3 aircraft. Both canards are lifting surfaces at the given flight conditions, thus suggesting it to be effective. A negative pressure can also be seen on the V-tail in configurations C-0, C-2 and C-3. The horizontal stabilizers in C-1 configuration are also lifting and thus able to provide nominal contribution to the aircraft longitudinal stability.

As the AoA is increased to $24^{\circ}$, the pressure distribution across the leading edge becomes nonuniform, which is evident from Fig. 13. The main reason is the magnification of wingtip vortices generated on the lower surface of the wing, which interacts with the flow over the upper surface and thus generating a span wise velocity component. Over the leading edges, $\mathrm{C}$-3 presents the best $\mathrm{C}_{\mathrm{p}}$ distribution, followed by $\mathrm{C}-0$ configuration. Concept- 1 configuration seems to be affected largely by wingtip vortices that can be attributed to its double delta configuration. Concept-3 also presents the best $\mathrm{C}_{\mathrm{p}}$ distribution over the wing surface. However, this is again undermined by the poor shape of blended nacelles, as high pressure is created at the front region (see Fig. 13). A negative pressure is maintained over the canards, and thus they are still effective at $24^{\circ}$ AoA. In terms of efficiency, C-2 canards appear to be more efficient as compared to C-3, but the difference is very small. Concept- 0 and C- 1 appear to have the best pressure distribution over the vertical tail. In C-3, a high-pressure region can be seen ahead of the V-tail, thus proposing an improvement in this region. Finally, there is a negative pressure over the horizontal stabilizer of $\mathrm{C}$-1, and thus it is contributing positively towards the stability of the aircraft. 

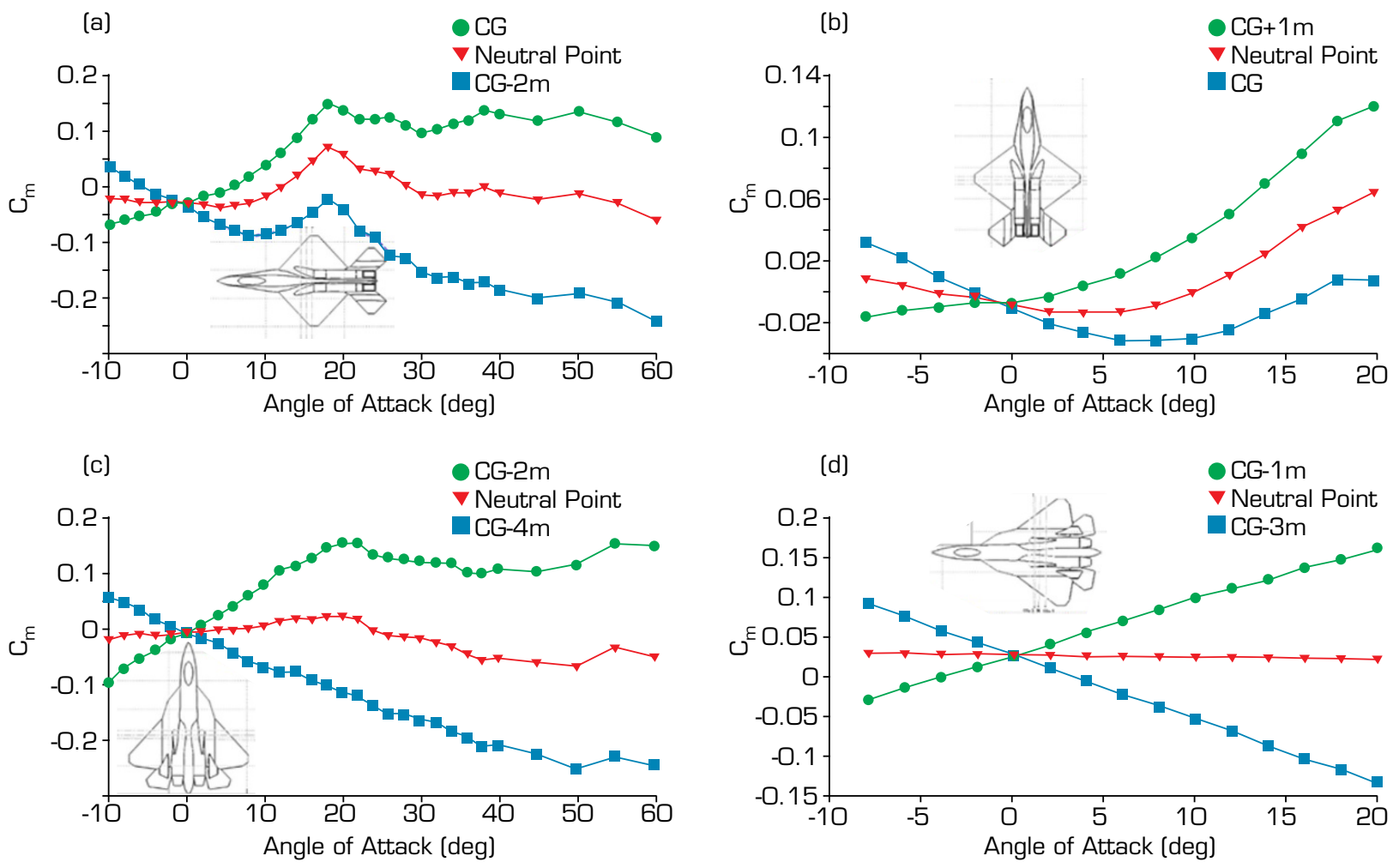

(d)

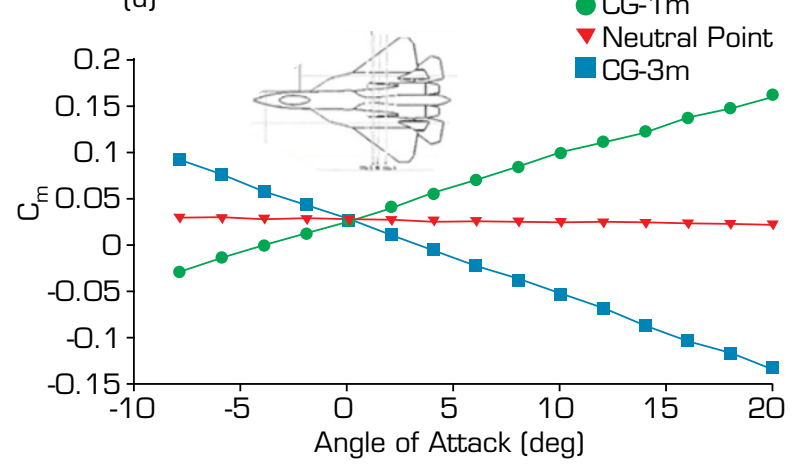

[e]
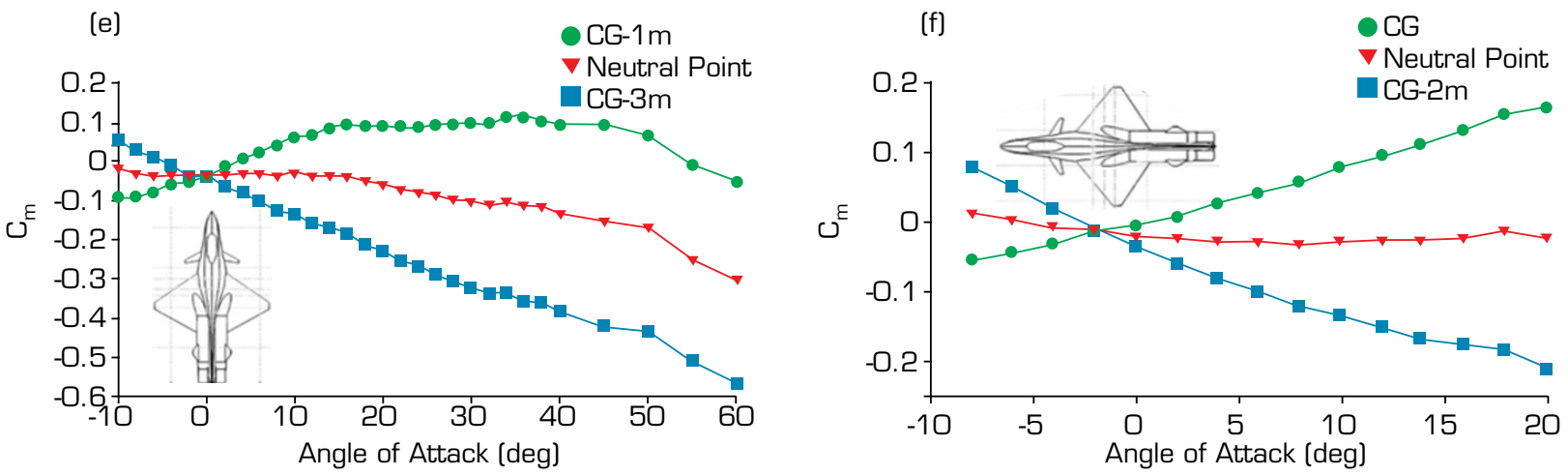

(g)

CG-2m
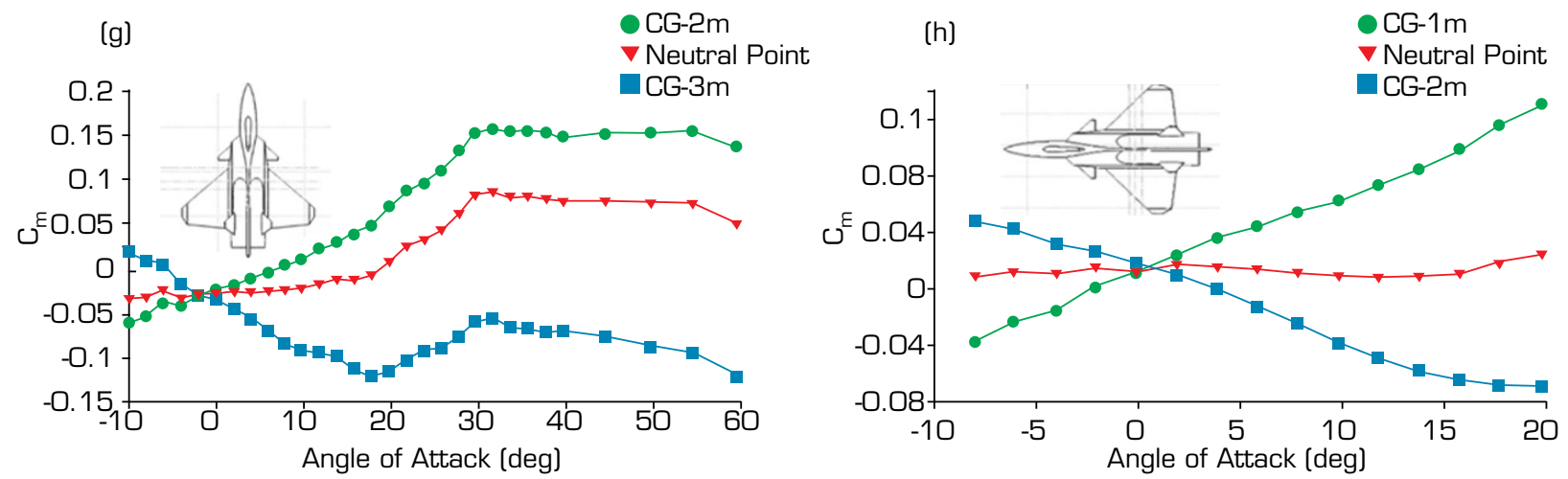

Figure 12. Pitching moment coefficient vs. AoA and Neutral Point estimation at subsonic and supersonic Mach. (a) Subsonic $C_{M}$ vs. AOA for C-0; (b) Supersonic $C_{M}$ vs. AOA for C-0; (c) Subsonic $C_{M}$ vs. AOA for C-1; (d) Supersonic $C_{M}$ vs. AOA for C-1; e) Subsonic $C_{M}$ vs. AOA for C-2; (f) Supersonic $C_{M}$ vs. AOA for C-2; (g) Subsonic $C_{M}$ vs. AOA for C-3; (h) Supersonic $C_{M}$ vs. AOA for C-3. 
(a)

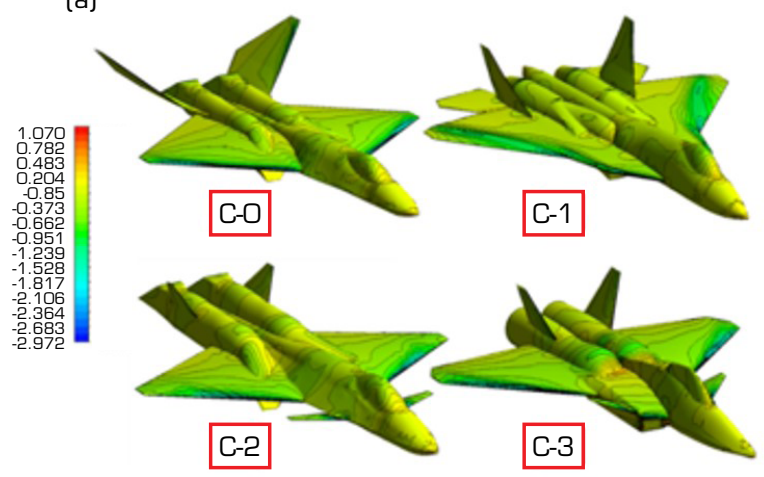

(c)

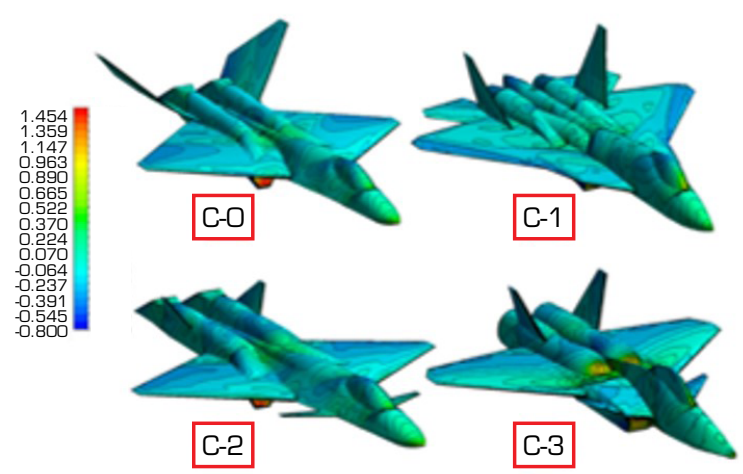

(b)

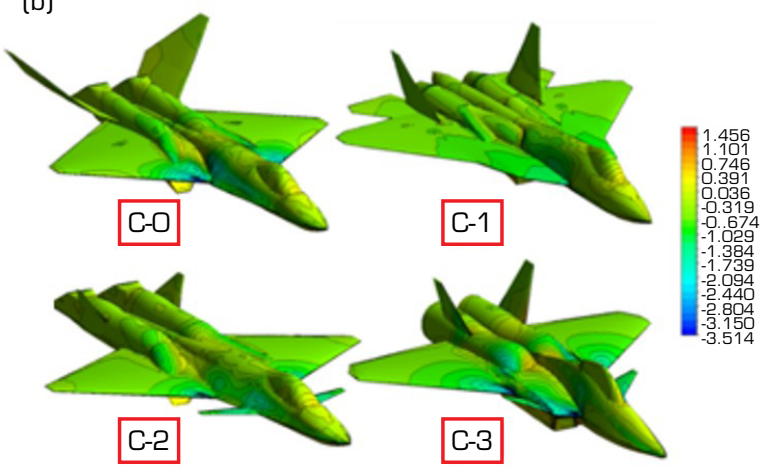

(d)

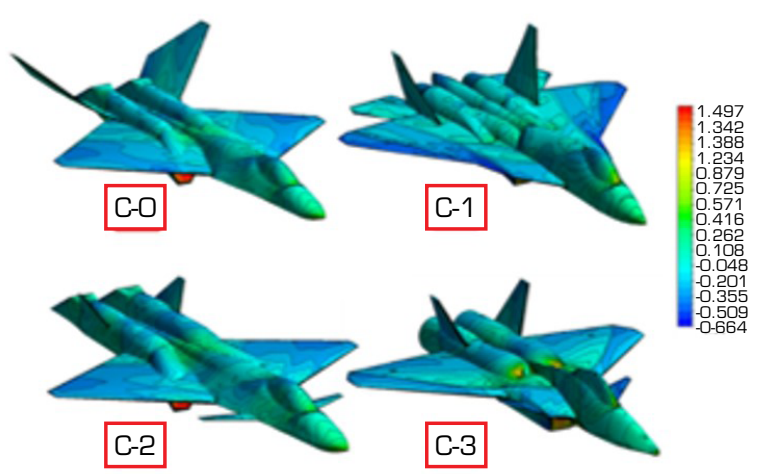

Figure 13. Comparison of Pressure coefficient at subsonic and supersonic Mach. (a) Subsonic $8^{\circ} \mathrm{AOA}$; (b) Subsonic $24^{\circ} \mathrm{AOA}$; (c) Supersonic $2^{\circ} \mathrm{AOA}$; (d) Supersonic $6^{\circ} \mathrm{AOA}$;

The shock waves are generated over the aircraft surface as flow becomes sonic, that is, $M \geq 1.0$. The primary implication of this shock is an increase in the static pressure of the air. This can be seen from the color legends in supersonic regime as the absolute value of negative pressure is dropped from 3 to 0.7. Figure 13 also presents the pressure distribution at supersonic speed at Mach 1.4 and $2^{\circ}$ AoA. The most impressive $\mathrm{C}_{\mathrm{p}}$ distribution over the leading edge is observed in $\mathrm{C}-1$ configuration. A large negative pressure can be seen over the wing surfaces of C-2 and C-3 configurations. The effect of poorly shaped blended nacelle is drastic at supersonic velocity as the flow is largely affected span wise over the C-3 wing. Concept- 0 wing also has appreciable lift distribution over the surface. Canard of C-3 has a larger sweep angle as compared to C-2 and thus appears to be more effective at supersonic speeds. Due to the formation of shock waves, a high pressure can also be seen over the leading edges of V-tail surface, that is, smoothened out as the flow moves downstream the surface. Large region of relative high pressure can also be seen over the V-tail of C-0 configuration. One of the notable features in C-3 configuration is the shape of its nose surface, which results in improved $\mathrm{C}_{\mathrm{p}}$ distribution. At supersonic state at $6^{\circ} \mathrm{AOA}$, it is evident that $\mathrm{C}-1$ has the best pressure distribution across the leading edges, followed by C- 3 configuration. Meanwhile, it is also improved on the leading edges of other configurations as well. Concept-1 has the best canopy shape due to its sleek design, the same was observed for all flight conditions. Moreover, C-1 appears to have the best exhaust design in terms of aerodynamic efficiency, as compared to other configurations for all simulated flight conditions. However, due to a sharp fairing and shock wave interaction, a slight high-pressure region can be observed in the mid fuselage section just ahead of blended engine nacelle. Regions of high pressure can also be seen in the mid fuselage region for different aircraft. The pressure distribution is slightly improved on the $\mathrm{V}$-tail of C-0 aircraft. Concept- 0 and C- 2 have the highest pressure at the engine inlets, while the lowest is seen on the C-1 configuration.

The eddies are the swirling of fluid that are generated when a turbulent flow passes an obstacle in its path. An eddy is not a property of fluid, but a violent swirling motion caused by the position and direction of turbulent flow. The eddy viscosity is the proportionality factor describing the turbulent transfer of energy as a result of moving eddies, thus giving rise to tangential stresses. 
At $10^{\circ} \mathrm{AoA}$, the small eddies are seen on the wing tips, indicating downwash from the wing. The eddies can also be seen on the downstream of the engine exhaust. As the AoA is increased, the magnitude of vortex shedding is also especially increased, which is visible in Fig. 14. At post-stall AoA, there is a decrement in the magnitude of eddy viscosity, primarily due to flow separation. At $50^{\circ}$, it is clear that the flow is separated completely from the surface of the aircraft.
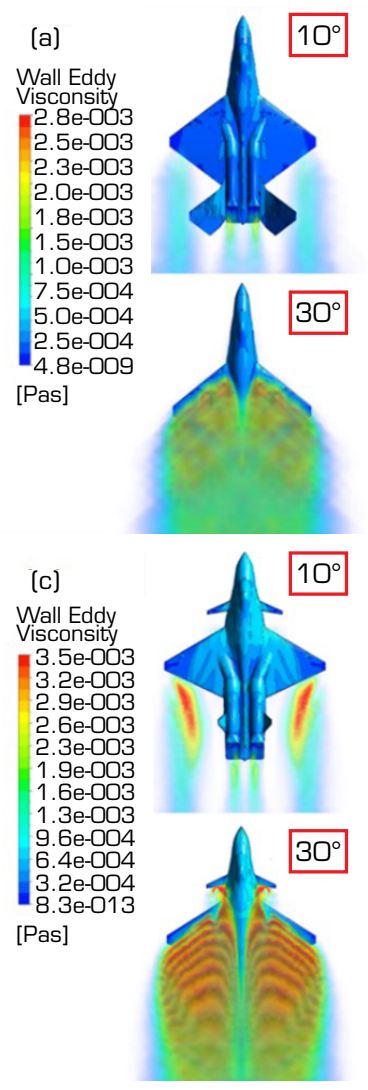
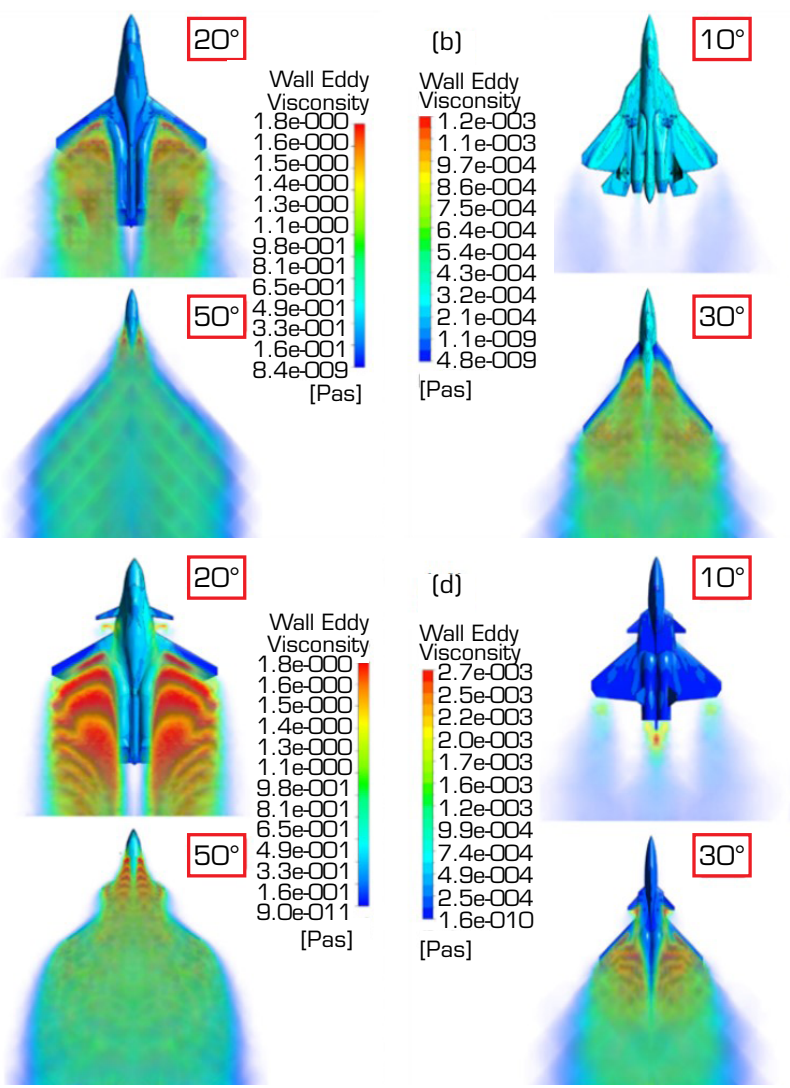

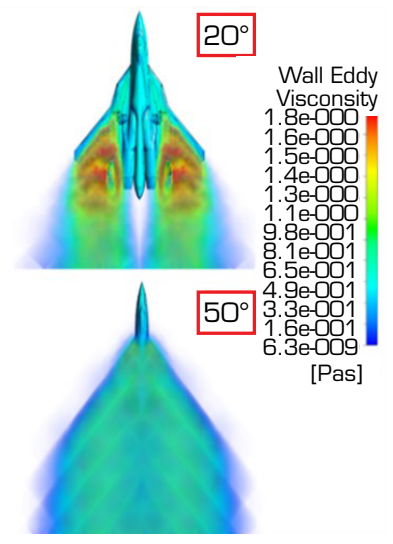

Figure 14. Visualization of eddy viscosity at various AoA. (a) Concept-0; (b) Concept-1; (c) Concept-2; (d) Concept-3.

Concept- 1 is found to have smallest eddies shedding from the wingtips at $10^{\circ} \mathrm{AoA}$ as compared to other configurations. The eddies are somewhat delayed and found to be emitting from the mid-chord wing surface at $20^{\circ}$ AoA. However, they are strong in magnitude and strongly affect the flow over the vertical tail. In the post-stall regime, Fig. 14 indicates the interaction among the vortices, especially in the reattachment of secondary wingtip vortices. At $50^{\circ}$, the aircraft appears to be stalled completely.

Concept-2 appeared to shed the strongest eddies than all other configurations. In Fig. 14, the strong eddies can be seen shedding from the wingtips at $10^{\circ} \mathrm{AoA}$. As the AoA is increased to $20^{\circ} \mathrm{AoA}$, the flow eddies grow in size and strength, producing at a location near to the quarter-chord of the wing. At $30^{\circ}$, the strength of eddy viscosity is somewhat reduced, except in the canard region, mainly due to the flow separation. At $50^{\circ}$, the strength of eddy viscosity is dissipated, except in the canard section.

The eddy viscosity is high at $10^{\circ} \mathrm{AoA}$ as compared to the other configurations, particularly in the engine exhaust region. The stronger eddies appear on the wing surface in the mid-chord region at $20^{\circ} \mathrm{AoA}$. The eddies become stronger as they travel along the surface and diminishes when moving away. Figure 14 shows that the $30^{\circ}$ is the post-stall regime as strength of eddies is decreased due to separation. At $50^{\circ}$, the flow appears to be separated completely on the aircraft, except on the nose section.

The flow topologies of the configurations at different AoA are illustrated by a common iso-value of Q-criterion in Fig. 15. Q-criterion is a flow visualization tool that highlights the vortex-based areas where the magnitude of the vorticity tensor $(\Omega)$ exceeds the magnitude of the strain rate tensor $(S)$ and is expressed by Eq. 2:

$$
Q=\frac{1}{2}\left(\|\Omega\|^{2}-\|\mathrm{S}\|^{2}\right)
$$


(a)
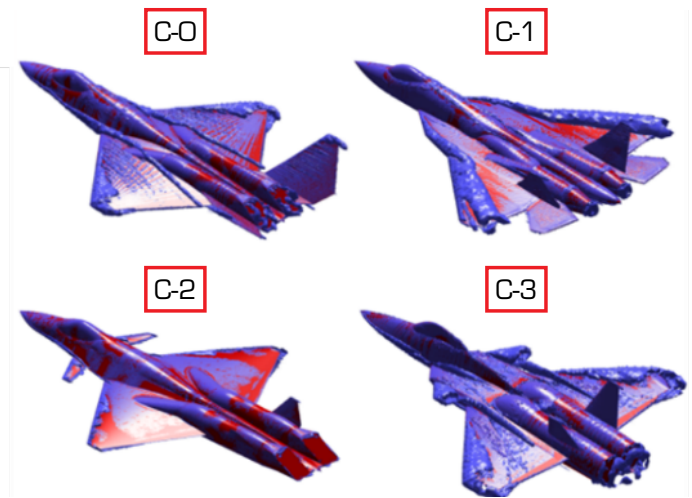

(c)
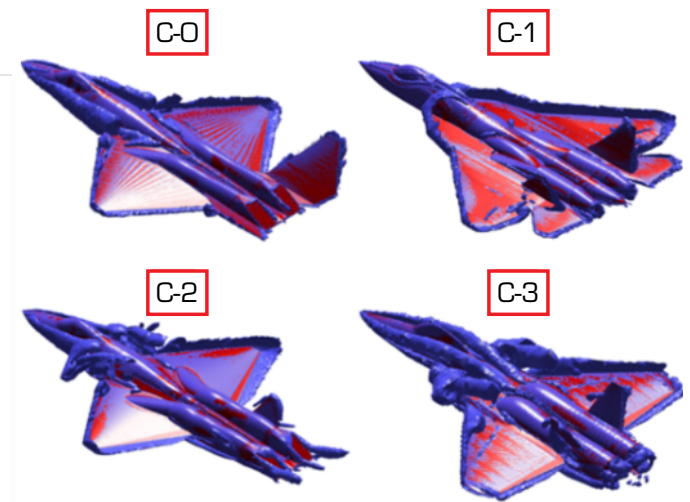

(b)
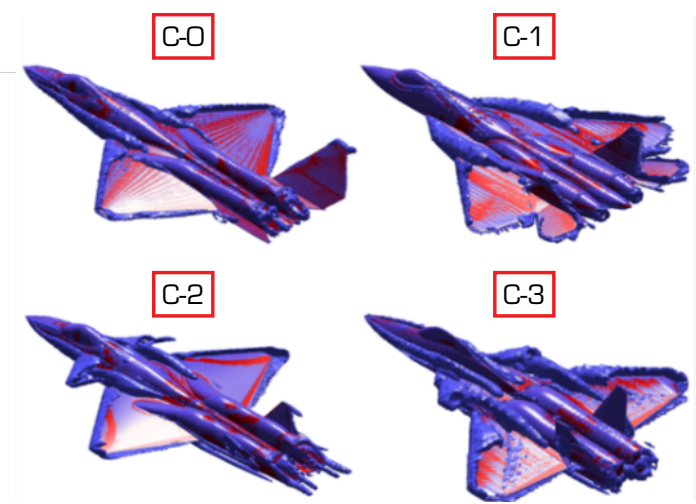

(d)
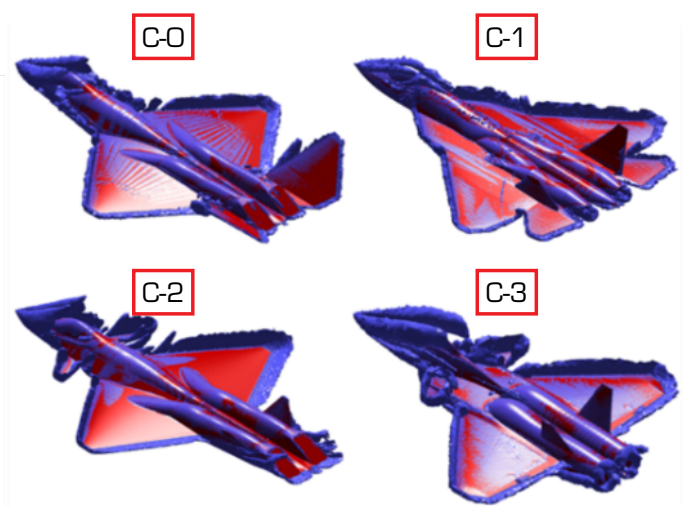

Figure 15. Visualization of the vortex core at a common value of $Q$ criterion at various flow angles. (a) $10^{\circ} \mathrm{AOA}$; (b) $20^{\circ} \mathrm{AOA}$; (c) $30^{\circ} \mathrm{AOA}$; (d) $50^{\circ} \mathrm{AOA}$.

From the figure, the major contribution of vortex generation at low to moderate AoA can be observed due to the pressure difference between upper and lower surfaces of the wing. The formation of forebody vortices, canard vortices and the wing tip and leading edges vortices majorly affects the aerodynamics of the configurations. At $10^{\circ}$ AoA (refer Fig. 15), the vortices of significant magnitude appear on the upper surfaces of C-1 and C-3. Concept-1 vortices are mainly due to its delta wing configuration, but are restricted to the wing tips only. The flow near the root chord of the wing seems to be unaffected as the flow moves along the surface. However, the vortices on C-3 indicate that it is disturbing the air flow on the surface of the wing because of canard surfaces. Concept- 0 and C-2 have smaller magnitude of vortices mainly on the edges of the surfaces. Concept- 2 has the minimum magnitude of vortices.

From Fig. 15, it can be observed that the vortices on the C- 0 and C- 2 remain small and restricted as compared to C- 1 and C-3 at $20^{\circ}$ AoA. However, canard of C-2 appears to be significantly affected by the vortices. Reynolds-averaged Navier-Stokes simulation has captured the interaction of canard, forebody and wing vortices in C-2 and C-3. Vortex core generated by the nose of the aircraft C- 0 and C- 2 moves downstream and ends near the quarter chord of the wing root. Concept-3 again has the largest magnitude of vortices. The vortices formed at the nose are larger in magnitude as compared to all other configurations. The vortices formed near the canard severely affect the canard surfaces and remain significant until the mid-chord of the wing root. The wing surface is also affected by the vortices, mainly at the leading edge, especially near the mean aerodynamic chord. There are no significant vortices near the nose of the C-1 aircraft even at $20^{\circ}$ AoA. The vortices are significant only at the leading edges of the front delta and weaken as it approaches to the quarter chord of the wing root. The vortices also diminishes as they travel downstream along the leading edges of the wing. The horizontal stabilizer is slightly affected by the vortices. The vortices also appear only on the junction of wing vertical tail, but they are smaller in magnitude due to small pressure difference.

At the post-stall $\mathrm{AoA}\left(50^{\circ}\right)$, it can be observed that C- 1 is relatively less affected by the vortices than the other configurations. Concept- 0 and C-2 have similar planforms and nose-shape, yet the effect of vortices is larger on C-2, especially in the trailing edge of wing. Canard surfaces of C-2 and C-3 are extremely affected at this AoA. Concept- 1 has a remarkable nose shape as the vortices are very low in magnitude, even at the post-stall AoA. There are significant vortices at the front delta wing, suggesting to be completely affected. At a very high AoA, Fig. 15 indicates that excessive vortices are formed in the nose region of C-0, C-2 and C-3 configuration with the highest for C-3. Concept-0 has 
less vortices along the edges of its wing as compared to other configurations, but still, they are substantial. The flow around the nose is found to be better for C-1 configuration due to its superior nose shape, yet, due to delta wing configuration, the wing is largely affected by the wing tip vortices. Concept-3 nose shape does not appear to be optimal, as it is generating larger region of vortices that indicates more drag.

\section{Leading-Edge Suction Analogy-Based Vortex Lift Estimation}

Based on the vortex lift concept using leading edge suction analogy, an empirical study is conducted for estimating the lift coefficient for delta wings at low AoA. The mathematical formulation of Polhamus method is expressed in Eq. 3:

$$
C_{L}=K_{P} \sin \alpha \cos ^{2} \alpha+K_{v} \cos \alpha \sin ^{2} \alpha
$$

where $K_{p}$ and $K_{v}$ are proportionality constant for potential and vortex lift. $K_{p}$ is dependent on the wing aspect ratio. On the contrary, the value $K_{v}$ is nearly constant and is usually taken as $\pi$.

The subject models have triangular wings with aspect ratios ranging from 2 to 2.6. Therefore, the force constants are evaluated through Eqs. 4 and 5, presented by Maybury et al. (2001):

$$
\begin{gathered}
K_{P}=1.393 A R-0.141 A R^{2} \\
K_{v}=3.157-0.020 A R+0.021 A R^{2}
\end{gathered}
$$

where AR represents the aspect ratio. The values of these constants are presented in Table 4 for each concept.

Table 4. Lift proportionality constants.

\begin{tabular}{rrrrrr}
\hline \multicolumn{2}{c}{ Concept-0 } & \multicolumn{2}{c}{ Concept-1 } & \multicolumn{2}{c}{ Concept-3 } \\
\hline AR & 2.0030 & AR & 2.3113 & AR & 2.5215 \\
\hline$K_{p}$ & 2.2245 & $K_{p}$ & 2.4664 & $K_{p}$ & 2.6160 \\
\hline$K_{v}$ & 3.2012 & $K_{v}$ & 3.2230 & $K_{v}$ & 3.2401 \\
\hline
\end{tabular}

The plots of the analytical and numerically computed value of the wing lift coefficients are depicted in Fig. 16 for each configuration. Among the given aircraft configuration, C-1 wing resembles the delta wing configuration and it can be observed from Fig. 16 that numerically calculated lift coefficient follows the analytical estimate curve closely for the given values of $K_{p}$ and $K_{v}$.

(a)
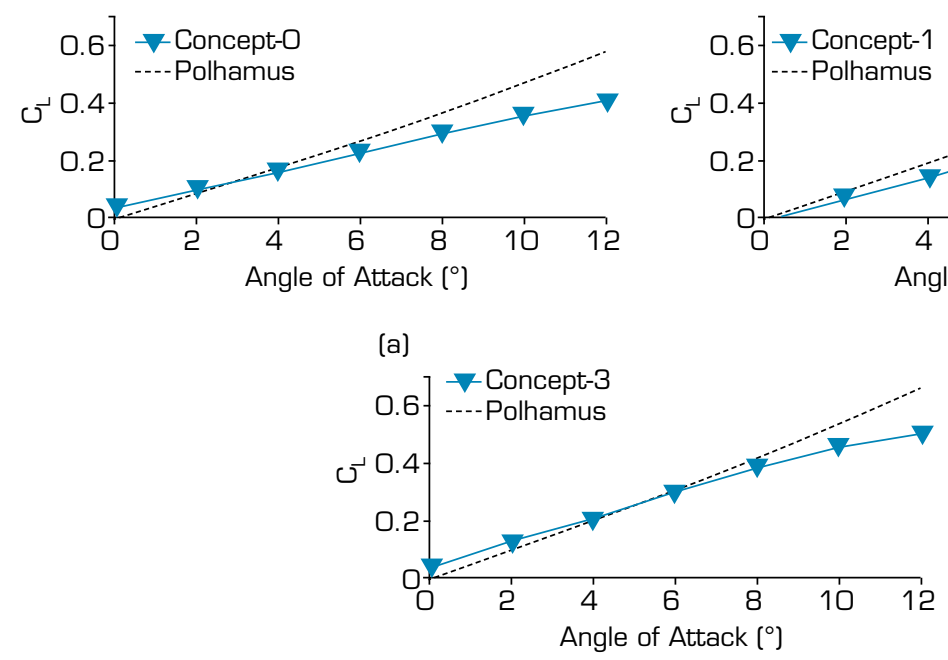

(a)

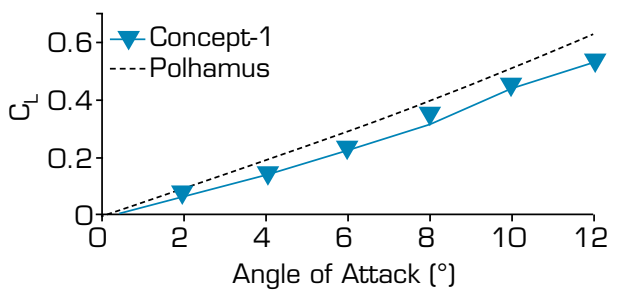

Figure 16. Comparison of analytical and numerical lift coefficient. (a) Concept-0; (b) Concept-1; (c) Concept-3. 


\section{Post-Stall Estimation of Lift Coefficient}

The numerical results are achieved till $60^{\circ}$, after which the solution largely oscillates and diverges. To predict the further behavior of the lift coefficient with variation in AoA, the curve fitting techniques were highlighted by Duquette (2007). Among these techniques, Duquette suggested the post-stall method by Viterra and Corrigan for accurate estimation of $\mathrm{C}_{\mathrm{L}}$ between the stall angle $\left(\alpha_{\text {stall }}\right)$ and $90^{\circ}$. Therefore, the method was implemented in the current research work, and Eqs. 6, 7, 8 and 9, presented in the research by Duquette (2007), were used.

$$
\begin{gathered}
C_{L}=A 1 \sin 2 \alpha+\frac{A 2 \cos ^{2} \alpha}{\sin \alpha} \\
A 1=\frac{C_{D, M A X}}{2} \\
A 2=\left(C_{L, \text { stall }}-C_{D, M A X} * \sin \alpha_{\text {stall }} * \cos \alpha_{\text {stall }}\right) \frac{\sin \alpha_{\text {stall }}}{\cos ^{2 \alpha_{\text {stall }}}} \\
C_{D, M A X}=1.11+0.018 A R
\end{gathered}
$$

Based on these formulations, Eqs. 10, 11, 12 and 13 were derived for the conceptual aircraft.

$$
\begin{aligned}
& C_{L}=0.57303 \sin 2 \alpha+\frac{0.97924 \cos ^{2} \alpha}{\sin \alpha} ;(C 0) \\
& C_{L}=0.57580 \sin 2 \alpha+\frac{0.91607 \cos ^{2} \alpha}{\sin \alpha} ;(C) \\
& C_{L}=0.57758 \sin 2 \alpha+\frac{0.96336 \cos ^{2} \alpha}{\sin \alpha} ;(C 2) \\
& C_{L}=0.57770 \sin 2 \alpha+\frac{0.94573 \cos ^{2} \alpha}{\sin \alpha} ;(C)
\end{aligned}
$$

It is notable to mention that these equations are applicable for the angles beyond $60^{\circ}$. The behavior of lift coefficient between the angles -10 and $90^{\circ}$ are presented in Fig. 17 for each configuration. This data is useful for modeling agile maneuvers of Advanced Tactical Fighter (ATF) for performance analysis and studying complex trajectories, such as cobra maneuvers.
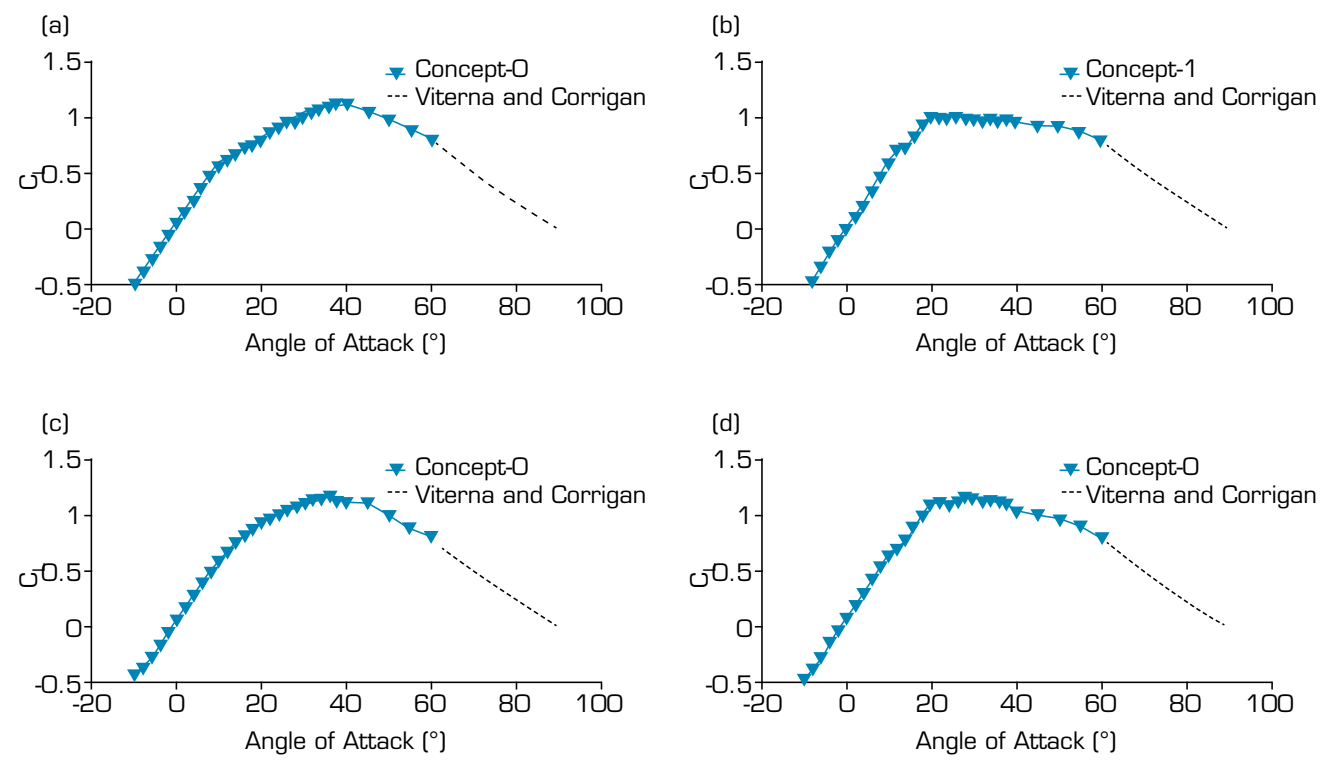

Figure 17. Lift coefficient trend based on CFD runs and post-stall method.

(a) Concept-0; (b) Concept-1; (c) Concept-2; (d) Concept-3. 


\section{Contribution of Components Fuselage}

Figure 18 shows the fuselage contribution towards lift, drag and lift-to-drag ratio at different AoA at a subsonic Mach No. of 0.6. Due to fairing on the C-0 configuration, lift on the fuselage region is found to be more as compared to other configurations. However, the drag encountered by it is also higher as compared to the other configuration at near and beyond stall AoA. This suggests an improvement in the geometry shape of fuselage in order to attain less drag and consequently a higher L/D ratio.
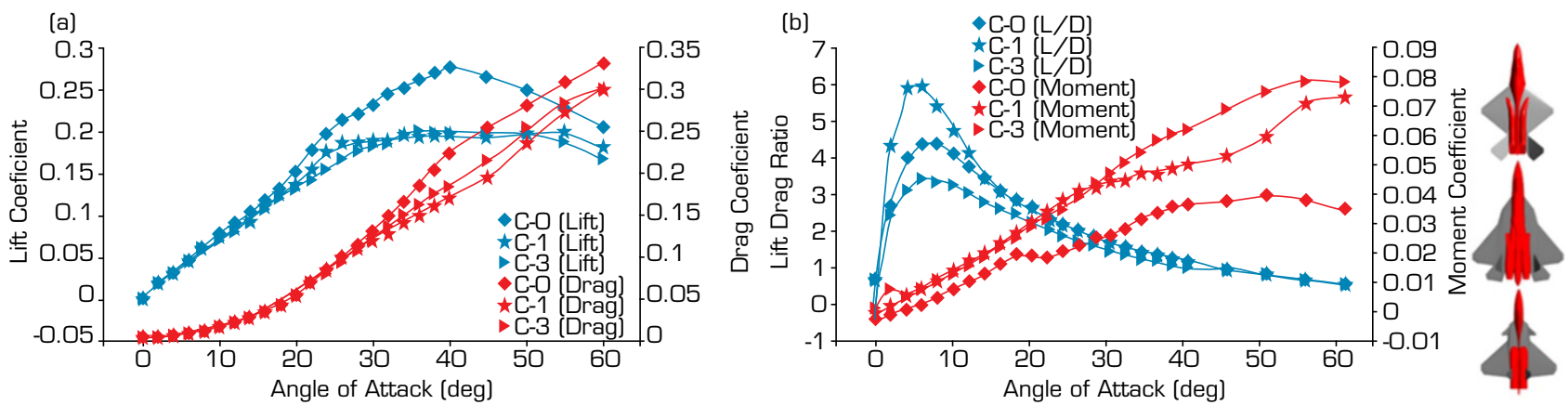

Figure 18. Aerodynamic contributions of Fuselage at Subsonic Mach. (a) Contribution in overall lift and drag. (b) Contribution in lift-to-drag ratio and pitching moment.

Concept-1 configuration has nominal lift at all AoA and has a relative less amount of drag. Better fairing on the surface and streamlined shape of the fuselage resulted in the highest $\mathrm{L} / \mathrm{D}$ among other configurations. Concept-3 has generated less lift due to the high-pressure region generated in the mid-fuselage section ahead of fairing. This has affected the aerodynamic performance, as $\mathrm{L} / \mathrm{D}$ is lower for C-3 at all AoA before stall.

\section{Wing}

The primary function of a wing is to generate sufficient lift in order to sustain flight and thus the most important component of an aircraft. The wing planform is the shape of the wing viewed directly from the above, coming in different configurations with each having its own advantages and disadvantages. Figure 19 compares the aerodynamic characteristics w.r.t AoA of the wing of different aircraft configurations in subsonic flight of Mach 0.6 . Concept- 0 configuration consist of a trapezoidal wing with a significant taper-ratio. The lift coefficient is found to be less than other aircraft at all AoA. However, as this configuration is much closer to the ideal elliptical wing, the drag coefficient is less than other aircraft, and lift-to-drag (L/D) ratio was highest for C-0 configuration. Concept- 0 configuration is marginally unstable as coefficient of moment increases slowly with AoA before stall. However, beyond stall it is found to be fairly stable. The stall is achieved at moderate AoA. After stall, the flow is found to remain attached only in the small portion of leading edge at $30^{\circ}$ AoA. At $50^{\circ}$, the flow is completely detached from the upper surface of the wing, leaving a large separation region suggesting a high pressure drag.
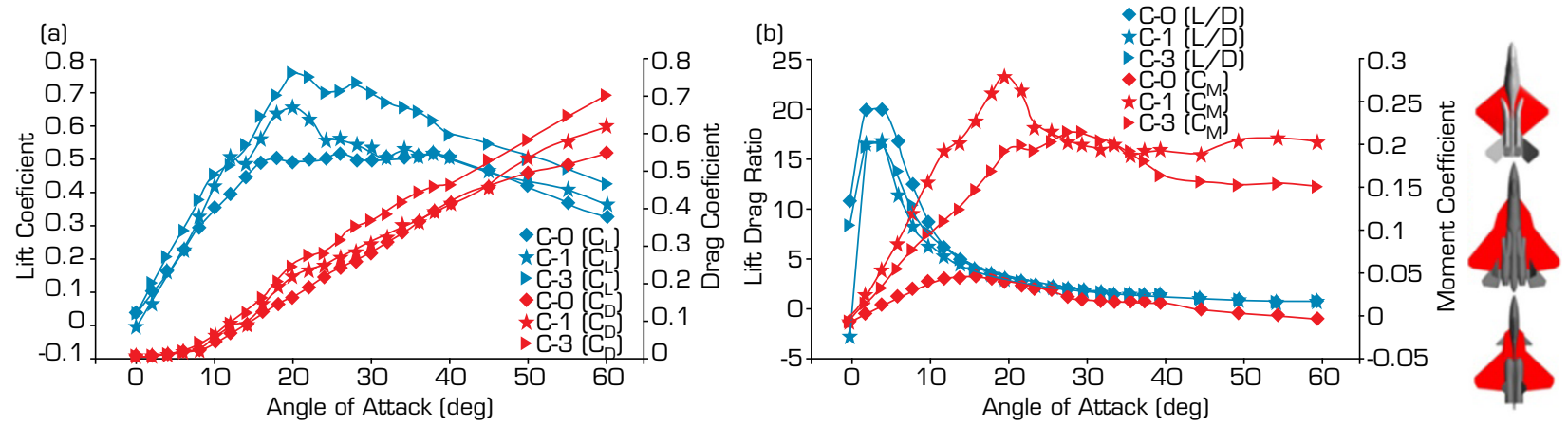

Figure 19. Aerodynamic contributions of Wing at Subsonic Mach. (a) Contribution in overall lift and drag. (b) Contribution in lift-to-drag ratio and pitching moment. 
In C-1 configuration, the wing planform is double-delta with sweep forward trailing edge. Lift coefficient is found to be more than C-0 configuration with relative higher drag. However, due to its delta wing configuration, stall is delayed to nearly $20^{\circ}$ AoA. As there is a rapid decrease in the lift coefficient, the post-stall effect is found to be dramatic. The lift-to-drag ratio is low at low AoA; however, it is improved as the AoA is increased and was found slightly higher than C-3. It can also be seen that the wing of $\mathrm{C}-1$ configuration is the most unstable configuration from the moment coefficient curve. Cm increases almost linearly with the AoA and results in higher slope than other configurations.

Concept-3 is a cropped delta wing aircraft. The lift coefficient is found higher for C-3 than all the other configurations throughout all AoA. However, the coefficient of drag can be observed to be higher as well. This decreases the overall aerodynamic performance of the wing as lift-to-drag ratio is not as impressive as C-0, but it is similar to C-1. The stall angle is slightly higher than C-1. Concept-3 wing is also unstable; however, the behavior is benign relative to C-1.

\section{Vertical Tail}

The tail in C-0 is in the form of V-tail with large surface region. In Fig. 20, the coefficient of lift is the highest, suggesting large nose-down moment being generated by the tail. This is true only till moderate AoA. The lift coefficient drops exponentially as the AoA is increased from $8^{\circ}$ and remains very low till $25^{\circ}$, signifying the inefficiency of tail in between these AoA. Since there is no horizontal stabilizer, a negative lift coefficient that can be seen in Fig. 20 further indicates a destabilizing nose-up moment being generated, which can also be seen in the right figure as an increase in the coefficient of moment. The lift-to-drag ratio is notable at low AoA. However, there is a sudden drop in L/D ratio as the AoA is in $20-25^{\circ}$ range. Concept- 1 has twin vertical tail, which is inclined at very small angle. The coefficient of lift is very small and slightly increases with the AoA. Nonetheless, it starts dropping at nearly $15^{\circ} \mathrm{AoA}$. In the post-stall regime, the coefficient of lift remains nearly constant throughout showing stabilizing effect on the aircraft. The coefficient of moment is marginally negative throughout all the AoA.
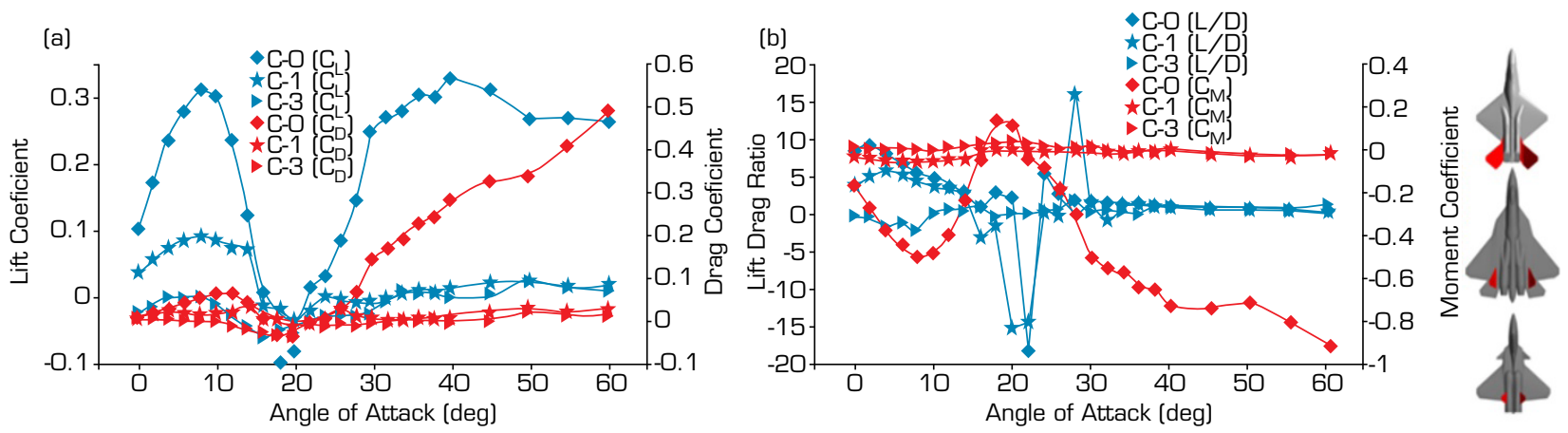

Figure 20. Aerodynamic contributions of Vertical tail at Subsonic Mach. (a) Contribution in overall lift and drag. (b) Contribution in lift-to-drag ratio and pitching moment.

The lift-to-drag ratio is impressive only at small AoA. There is a drop in $\mathrm{L} / \mathrm{D}$ ratio to negative values as the AoA is between $15-25^{\circ}$ range. There is a V-tail configuration in C-3 configuration, which is smaller in size. There is no horizontal stabilizer present in the aircraft. The lift-coefficient is small and mostly negative before stall. The coefficient of moment is slightly negative throughout all AoA. The lift-to-drag ratio shows poor aerodynamic efficiency of the vertical tail with highest value of 2 2.5. Overall, the liftto-drag ratio is not impressive at all AoA, which indicates the need for modification in the design of V-tail of the C-3 aircraft.

\section{Canard/Horizontal Tail}

Figure 21 depicts the aerodynamic characteristics of horizontal surfaces of C-1 and C-3 configurations. A sweepback canard is present in C-3 configuration ahead of the wing. Canards are located ahead of the CG intentionally in order to destabilize the aircraft for greater maneuverability, especially at high AoA. From the $\mathrm{C}_{\mathrm{L}-\alpha}$ curve, the canard is seen to be generating lift efficiently till $30^{\circ}$ AoA. As the angle is increased from $30^{\circ}$, the lift coefficient starts to drop, suggesting it to be stalled. The lift-to-drag ratio is very high at low AoA, reaching a value of 28 before dropping at moderate AoA. The canards are found to have positive moment 
coefficient through all the AoA. The horizontal tail is present in C-1 configuration only just aft of the wing. The primary role of the horizontal tail is to provide longitudinal stability to the aircraft. At low AoA, the lift coefficient of horizontal tail is not notable, thus indicating poor performance at those angles. At moderate to high AoA, there is a sudden increase in the lift coefficient before retaining a nearly constant value. Moment coefficient is found to be very small and slightly negative at moderate-high AoA. The liftto-drag ratio is not very impressive as well. A redesign of the horizontal stabilizer is suggested to ensure better performance under vortex shedding from the wing.
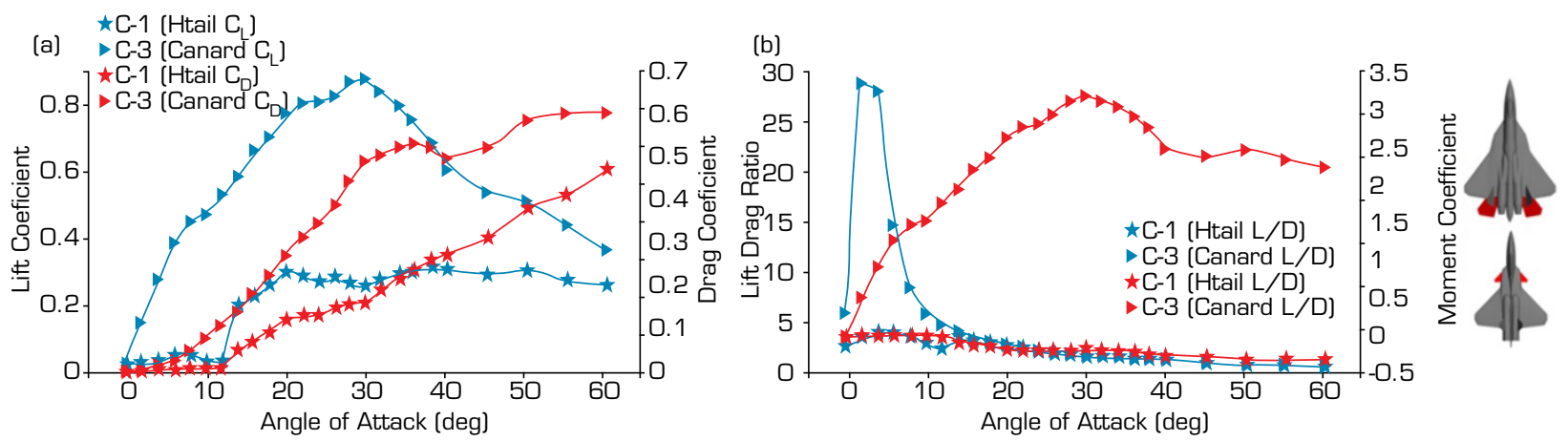

Figure 21. Aerodynamic contributions of Horizontal Tail / Canard at Subsonic Mach. (a) Contribution in overall lift and drag. (b) Contribution in lift-to-drag ratio and pitching moment.

\section{CONCLUSION}

Computational fluid dynamics is known to be a very useful tool in the aerodynamic analysis of aircraft. It not only provides deep information about the flow, which helps in the design optimization, but is also very cost-effective as compared to experimental techniques. However, for complex analyses, computational cost increases exponentially, yet significant less than wind tunnel operational cost.

Due to the unavailability of aerodynamic data on the fighter aircraft at different Mach numbers, CFD model was validated against ONERA M6 wing and UCAV SACCON, which provides fairly accurate results for 3D flows. However, many complex flow phenomena, like interaction of downstream vortices with the surface, post-stall aerodynamics, interference etc., need better benchmark for the CFD validation. In order to gain confidence in the CFD model, mesh sensitivity is checked and tight convergence criteria is employed in order to have minimum numerical errors. Computational fluid dynamics analysis provides key aerodynamic performance and stability parameters about the four conceptual designs (C-0, C-1, C-2 and C-3).

The comparison of aerodynamic performance parameter, which is a ratio of the lift-to-drag (L/D) of different aircraft in subsonic and supersonic flight regimes, has been studied and presented in this study. The highest wave drag is observed for the configuration C-0 wing to the abrupt changes in area distribution. However, the latter concept is the only among the four models symbolizing a positive static margin based on the CG location. Concept-1 performs the best in terms of drag behavior and lift to drag ratio in both flow regimes. Additionally, the highest supersonic lift and drag are observed in the configuration C-2. In subsonic regime, all configurations are longitudinally statically unstable. By carefully analyzing the shift of neutral point in moving from subsonic to supersonic flight regime, the aircraft becomes more statically stable. Also, each configuration exhibits flat surface behavior of $\mathrm{C}_{\mathrm{L}}$ at very high AoA.

Furthermore, the areas in which improvements can be made in order to further enhance its flight capability were also identified from the flow visualization over the four aircraft. It is highly recommended to add engine fairing in C-3 concept and repositioning of its canard surface, so that the effects of vortices on the wings are minimized. The reshaping of the canopy should also be considered. Concept-1 fuselage appears to have slightly better aerodynamic features, as it shows higher values of L/D at normal flying AoA. Concept-1 seems to have lowest drag in the supersonic regime, yet it is recommended to redesign blended engine nacelle. The features that are suggested to be improved in C-0 are wing leading edge, engine nacelle fairing and vertical tail. 


\section{AUTHORS' CONTRIBUTION}

Conceptualization: Siddiqui W, Maqsood A, Riaz R, Salamat S; Methodology: Siddiqui W, Zahid M.S, Naseer H, Maqsood A; Investigation: Siddiqui W, Zahid M.S, Naseer H; Writing - Original Draft: Siddiqui W and Maqsood A; Writing - Review and Editing: Siddiqui W and Maqsood A; Supervision: Maqsood A, Riaz R and Salamat S.

\section{DATA AVAILABILITY STATEMENT}

All the datasets were generated and analyzed during the current study.

\section{FUNDING}

Not applicable.

\section{ACKNOWLEDGEMENTS}

The authors would like to extend their sincere thanks to the supercomputing facility at Research Center for Modelling and Simulation for their technical support.

\section{REFERENCES}

Abney E, McDaniel M (2005) High Angle of Attack Aerodynamic Predictions Using Missile Datcom. Paper presented 23rd AIAA Applied Aerodynamics Conference. AIAA; Toronto, Ontario, Canada. https://doi.org/10.2514/6.2005-5086

Amadori K, Jouannet C, Krus P (2008) Aircraft Conceptual Design Optimization. Paper presented ICAS 26th International Congress of the Aeronautical Sciences.

Sutrisno S, Wibowo SB, Iswahyudi S, Rohmat TA (2019) Rolled-Up Vortex Dynamic Numerical Studies of Bird's-Body-Type Fighter. Int J Mech Eng Technol 10(3):304-316.

Bitencourt LO, Pogorzelski G, Freitas RM, Azevedo JLF (2011) A CFD-based analysis of the 14-Bis aircraft aerodynamics and stability. J Aerosp Technol Manag 3(2):137-146. https://doi.org/10.5028/jatm.2011.03021711

Blake W (1985) Prediction of fighter aircraft dynamic derivatives using Digital Datcom. Paper presented 3rd Applied Aerodynamics Conference. AIAA; Colorado Springs, Colorado, United States. https://doi.org/10.2514/6.1985-4070

Boelens OJ (2012) CFD analysis of the flow around the X-31 aircraft at high angle of attack. Aerosp Sci Technol 20(1):38-51. https://doi.org/10.1016/j.ast.2012.03.003

Della Vecchia P, Ciliberti D (2013) Numerical aerodynamic analysis on a trapezoidal wing with high lift devices: a comparison with experimental data. Paper presented XXII AIDAA Conference. AIDAA; Naples, Italy. 
Duquette M (2007) Development and Application of SimpleFlight, a Variable-Fidelity Flight Dynamics Model. Paper presented AIAA Modeling and Simulation Technologies Conference and Exhibit. AIAA; Hilton Head, South Carolina, United States. https://doi.org/10.2514/6.2007-6372

ANSYS Inc (2017) ANSYS Fluent Theory Guide. Release 18.0. ANSYS Inc.

Kostić IA, Stefanović ZA, Kostić OP (2014) Aerodynamic analysis of a light aircraft at different design stages. FME Trans 42:94-105.

Kryvokhatko IS, Masko OM (2017) Aerodynamic Characteristics and Longitudinal Stability of Tube Launched TandemScheme UAV. In Volkov K, editor. Flight Physics: Models, Techniques and Technologies. London: IntechOpen. p. 73-83.

Loeser T, Vicroy D, Schuette A (2010) SACCON Static Wind Tunnel Tests at DNW-NWB and 14'x22' NASA LaRC. Paper presented 28th AIAA Applied Aerodynamics Conference. AIAA; Chicago, Illinois, United States. https://doi. org/10.2514/6.2010-4393

López D, Domínguez D, Gonzalo J (2013) Impact of turbulence modelling on external supersonic flow field simulations in rocket aerodynamics. Int J Comut Fluid Dyn 27(8-10):332-341. https://doi.org/10.1080/10618562.2013.867951

Mason W, Knill D, Giunta A, Grossman B, Watson L, Haftka R (1998) Getting the full benefits of CFD in conceptual design. Paper presented 16th AIAA Applied Aerodynamics Conference. AIAA; New Mexico, United States. https://doi. org/10.2514/6.1998-2513

Maybury WJ, Rayner JMV, Couldrick LB (2001) Lift generation by the avian tail. Proc R Soc Lond B Biol Sci 268(1475):14431448. https://doi.org/10.1098/rspb.2001.1666

Menter FR (1994) Two-equation eddy-viscosity turbulence models for engineering applications. AIAA J 32(8):1598-1605. https://doi.org/10.2514/3.12149

Nelson RC (1998) Flight Stability and Automatic Control. New York: McGraw-Hill Education.

Nicolosi F, Della Vecchia P, Ciliberti D, Cusati V (2014) Development of new preliminary design methodologies for regional turboprop aircraft by CFD analyses. 29th Congress of the International Council of the Aeronautical Sciences. St. Petersburg, Russia.

Schminder J (2012) Feasibility study of different methods for the use in aircraft conceptual design. Linköpings (Thesis). Linköpings Universitetet. In english.

Schmitt V, Charpin F (1979) Pressure Distributions on the ONERA M6-Wing at Transonic Mach Numbers. In AGARD, editor. Experimental Data Base for Computer Program Assessment. Seine: AGARD.

Schütte A, Cummings RM, Loeser T, Vicroy DD (2009) Integrated Computational/Experimental Approach to UCAV and Delta-Canard Configurations Regarding Stability \& Control. Paper presented 4th Symposium on Integrating CFD and Experiments in Aerodynamic. Brüssel, Belgien.

Spalart P, Allmaras S (1992) A one-equation turbulence model for aerodynamic flows. Paper presented 30th Aerospace Sciences Meeting and Exhibit. AIAA; Reno, Nevada, United States. https://doi.org/10.2514/6.1992-439

Tinoco EN (1991) CFD codes and applications at Boeing. Sadhana 16(Part 2):141-163. https://doi.org/10.1007/BF02812178

Tseng JB, Lan CE (1988) Calculation of Aerodynamic Characteristics of Airplane configurations at High Angles of Attack. Washington: NASA.

Wibowo SB, Sutrisno I, Rohmat TA (2018) An Evaluation of Turbulence Model for Vortex Breakdown Detection Over Delta Wing. Arch Mech Eng 65(3):399-415. 
Williams JE, Vukelich SR (1979) The USAF Stability and Control Digital DATCOM. Volume I, Users Manual. St. Louis: McDouglas Astronautics Company.

Xue RR, Ye ZY, Wang G (2016) Aerodynamic Characteristic Comparison of the Forward and Backward-Swept Wings. Paper presented 30th Congress of the International Council of the Aeronautical Sciences. ICAS; Daejeon, Korea.

Zhong Y, Zhao K (2012) Cfd-based research of strake effects for low speed high lift configuration. Paper presented 28th International Congress of the Aeronautical Sciences. ICAS; Brisbane, Australia. 